\title{
MIPAS-STR measurements in the Arctic UTLS in winter/spring 2010: instrument characterization, retrieval and validation
}

\author{
W. Woiwode ${ }^{1}$, H. Oelhaf ${ }^{1}$, T. Gulde ${ }^{1}$, C. Piesch ${ }^{1}$, G. Maucher ${ }^{1}$, A. Ebersoldt ${ }^{2}$, C. Keim ${ }^{1, *}$, M. Höpfner ${ }^{1}$, S. Khaykin ${ }^{3}$, \\ F. Ravegnani ${ }^{4}$, A. E. Ulanovsky ${ }^{3}$, C. M. Volk ${ }^{5}$, E. Hösen ${ }^{5}$, A. Dörnbrack ${ }^{6}$, J. Ungermann ${ }^{7}$, C. Kalicinsky ${ }^{5}$, and \\ J. Orphal ${ }^{1}$ \\ ${ }^{1}$ Institute for Meteorology and Climate Research, Karlsruhe Institute of Technology, Karlsruhe, Germany \\ ${ }^{2}$ Institute for Data Processing and Electronics, Karlsruhe Institute of Technology, Karlsruhe, Germany \\ ${ }^{3}$ Central Aerological Observatory, Dolgoprudny, Moscow region, Russia \\ ${ }^{4}$ Institute of Atmospheric Sciences and Climate, CNR-ISAC, Bologna, Italy \\ ${ }^{5}$ Department of Physics, University of Wuppertal, Wuppertal, Germany \\ ${ }^{6}$ DLR, Institut für Physik der Atmosphäre, 82230 Oberpfaffenhofen, Germany \\ ${ }^{7}$ Institute of Energy and Climate Research, Research Centre Jülich GmbH, Jülich, Germany \\ "now at: Astrium GmbH, Friedrichshafen, Germany
}

Correspondence to: W. Woiwode (wolfgang.woiwode@ kit.edu)

Received: 7 November 2011 - Published in Atmos. Meas. Tech. Discuss.: 24 November 2011

Revised: 24 April 2012 - Accepted: 8 May 2012 - Published: 1 June 2012

\begin{abstract}
The mid-infrared FTIR-limb-sounder Michelson Interferometer for Passive Atmospheric SoundingSTRatospheric aircraft (MIPAS-STR) was deployed onboard the research aircraft M55 Geophysica during the RECONCILE campaign (Reconciliation of Essential Process Parameters for an Enhanced Predictability of Arctic Stratospheric Ozone Loss and its Climate Interactions) in the Arctic winter/spring 2010. From the MIPAS-STR measurements, vertical profiles and 2-dimensional vertical cross-sections of temperature and trace gases are retrieved. Detailed mesoscale structures of polar vortex air, extra vortex air and vortex filaments are identified in the results at typical vertical resolutions of 1 to $2 \mathrm{~km}$ and typical horizontal sampling densities of 45 or $25 \mathrm{~km}$, depending on the sampling programme. Results are shown for the RECONCILE flight 11 on 2 March 2010 and are validated with collocated in-situ measurements of temperature, $\mathrm{O}_{3}$, CFC-11, CFC-12 and $\mathrm{H}_{2} \mathrm{O}$. Exceptional agreement is found for the in-situ comparisons of temperature and $\mathrm{O}_{3}$, with mean differences (vertical profile/along flight track) of $0.2 /-0.2 \mathrm{~K}$ for temperature and $-0.01 / 0.05$ ppmv for $\mathrm{O}_{3}$ and corresponding sample standard deviations of the mean differences of $0.7 / 0.6 \mathrm{~K}$ and $0.1 / 0.3 \mathrm{ppmv}$. The comparison of the retrieved vertical crosssections of $\mathrm{HNO}_{3}$ from MIPAS-STR and the infrared limb-
\end{abstract}

sounder Cryogenic Infrared Spectrometers and Telescopes for the Atmosphere-New Frontiers (CRISTA-NF) indicates a high degree of agreement. We discuss MIPAS-STR in its current configuration, the spectral and radiometric calibration of the measurements and the retrieval of atmospheric parameters from the spectra. The MIPAS-STR measurements are significantly affected by continuum-like contributions, which are attributed to background aerosol and broad spectral signatures from interfering trace gases, and are important for mid-infrared limb-sounding in the Upper Troposphere/Lower Stratosphere (UTLS) region. Taking into consideration continuum-like effects, we present a scheme suitable for accurate retrievals of temperature and an extended set of trace gases, including the correction of a systematic line-of-sight offset.

\section{Introduction}

Airborne and balloon-borne remote-sensing measurements are filling the gap between in-situ measurements and satellite-borne remote-sensing measurements in terms of spatial coverage and spatial resolution. While classical insitu instruments onboard aircraft or balloon platforms allow 
for measurements with high absolute accuracy constrained to the flight track, satellite remote measurements allow for maximal vertical sampling range, global coverage and extended time series, but often with higher uncertainties. In contrast to comparable satellite techniques, remote-sensing measurements from aircraft and balloon platforms focus on limited atmospheric areas, but with a much higher sampling density. The proximity of the sampled air-masses results in lower uncertainties in the retrievals, since pointing errors, non-local thermodynamic equilibrium effects in the spectra and effects from extended horizontal trace gas gradients are less severe. Further advantages of airborne high-altitude remote-sensing measurements are that flight scenarios can be adapted to scientific objectives individually and specific atmospheric structures can be targeted flexibly.

The FTIR limb-sounder MIPAS-STR (Piesch et al., 1996) onboard the high altitude aircraft M55 Geophysica is the airborne version of the series of MIPAS-instruments (Fischer and Oelhaf, 1996), including also balloon-borne (FriedlVallon et al., 2004) and satellite-borne techniques (Fischer et al., 2008). From the limb-emission measurements of MIPASSTR, vertical profiles and two-dimensional cross-sections of atmospheric temperature and trace gases $\left(\mathrm{HNO}_{3}, \mathrm{O}_{3}, \mathrm{CFCs}\right.$, $\mathrm{ClONO}_{2}, \mathrm{H}_{2} \mathrm{O}$ and several other species) are derived, allowing for reconstructing "snapshots" of the chemical and dynamical atmospheric situation along the flight-track.

MIPAS-STR was first deployed during the Antarctic campaign APE-GAIA (Airborne Polar ExperimentGeophysica Aircraft In Antarctica) in 1999 (Höpfner et al., 2000). In the following years, MIPAS-STR was operated in several other scientific campaigns and was applied for the validation of MIPAS-ENVISAT (Blom et al., 2004; Keim et al., 2004; Cortesi et al., 2007; Höpfner et al., 2007; Wang et al., 2007) and the microwave limbsounder MARSCHALS (Millimetre-wave Airborne Receiver for Spectroscopic CHaracterisation of Atmospheric LimbSounding) (Dinelli et al., 2009). The vertical profile of peroxyacetyl nitrate (PAN) in the upper tropical troposphere was derived from measurements of MIPAS-STR (Keim et al., 2008). MIPAS-STR was recently operated during the RECONCILE campaign (Reconciliation of Essential Process Parameters for an Enhanced Predictability of Arctic Stratospheric Ozone Loss and its Climate Interactions, see https: //www.fp7-reconcile.eu) in the Arctic Winter 2009/2010.

We describe the instrument in its current configuration and present results for RECONCILE flight 11 on 2 March 2010. An advanced retrieval approach is established considering continuum-like effects which significantly affect midinfrared limb-emission spectra in the altitude-range sampled by MIPAS-STR. Retrieval results are shown for temperature and an extended set of trace gases. The results of MIPASSTR are compared with results from in-situ instruments and the mid-infrared limb-sounder CRISTA-NF, which were deployed onboard the Geophyisca simultaneously.
An overview of MIPAS-STR in its actual setup is given in Sect. 2 and the sampling characteristics are discussed in Sect. 3. The spectral and radiometric calibration scheme is summarized in Sect. 4. The atmospheric situation and the applied sampling strategy during RECONCILE flight 11, as well as the cloud detection procedure from MIPAS-STR spectra are discussed in Sect. 5. Aspects of the retrieval are described in Sect. 6. The results and validation are discussed in Sect. 7 and conclusions are drawn in Sect. 8.

\section{Instrument overview}

MIPAS-STR is a cryogenic high-resolution FTIRspectrometer capable of detecting the limb-emission spectra of atmospheric trace gases in the mid-infrared. The instrument is described in its previous configurations in Piesch et al. (1996), Kimmig (2001) and Keim (2002). Many aspects of the instrument are comparable to the balloon-borne instrument MIPAS-B2 (Friedl-Vallon et al., 2004) and to a certain extent to the satellite-borne instrument MIPAS-ENVISAT (Fischer et al., 2008). Here, we give an updated summary of the instrument characteristics.

The instrument is deployed onboard the high-altitude aircraft M55 Geophysica, allowing for flight altitudes of up to $20 \mathrm{~km}$ and operating ranges of around $3000 \mathrm{~km}$ at a typical airspeed of 700 to $750 \mathrm{~km} \mathrm{~h}^{-1}$. MIPAS-STR is pointing towards the right hand side of the flight path (perpendicular relative to nose of aircraft). Sampling is performed in limb-mode and upward-looking geometries, allowing for the subsequent reconstruction of vertical profiles and twodimensional cross-sections of temperature and trace gases.

The instrument is set up by the optics module, including the scan-mirror, telescope, interferometer and detector unit, and the electronics module, including the data-processing and instrument-control electronics. A schematic representation of the optics module is shown in Fig. 1 and the characteristics of the instrument in the current setup are summarized in Table 1.

Infrared radiation from the probed airmass entering the instrument is directed via the scan-mirror into the telescope, subsequently the interferometer and the detector dewar. The main function of the 3-mirror telescope with an optical conversion ratio of 1.7 is the suppression of radiation from outside the field-of-view (FOV), which is scattered at surfaces inside the instrument or is diffracted at the edges of the front optics. The vertical FOV weighting function is characterized by calibration measurements on ground to minimize uncertainties in the retrievals. Instrumental line shape (ILS) related effects on the retrieval are considered by a theoretical model (Stiller et al., 2000).

The effective optical path difference of the double pendulum interferometer (Fischer and Oelhaf, 1996) is $\pm 13.9 \mathrm{~cm}$. Two-sided interferograms are recorded, resulting in spectra with an unapodized spectral resolution of $0.036 \mathrm{~cm}^{-1}$. After 


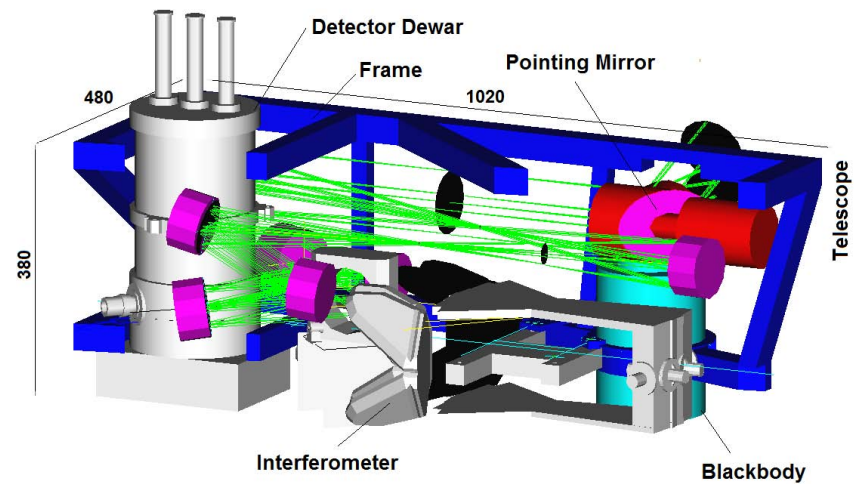

Fig. 1. Schematic representation of the optics module of MIPASSTR (Blom et al., 1998). Mirrors are shown in pink and the light path along scan-mirror, telescope and interferometer towards the detector dewar is indicated by green lines. Dimensions indicated in $\mathrm{mm}$.

applying the Norton-Beer strong apodization (Norton and Beer, 1976), an effective spectral resolution of $0.058 \mathrm{~cm}^{-1}$ is obtained. The effects of vibrations, which are typical for an aircraft platform like the Geophyisca, on the interferometer of MIPAS-STR have been investigated by Kimmig (2001). A dedicated time-equidistant sampling method introduced by Brault (1996) was adapted for the recording of interferograms such that perturbations in the spectra resulting from vibrations are minimized.

In order to minimize instrumental background radiation, the instrument is dry ice-cooled to about $210 \mathrm{~K}$ and the different reflective optics are coated with gold, protected gold or Silflex MK2 $2^{\mathrm{TM}}$. The detector is cooled by liquid helium to about $4 \mathrm{~K}$. Incoming infrared radiation enters the detector dewar via an anti-reflectance coated $\mathrm{ZnSe}$ window. Inside the detector dewar, the radiation is split up into four parts by dichroics and is directed via filters and blockers to the Si:As back-illuminated band-impurity detectors of the four channels. In this context, results from the spectral channel 1 (725 to $990 \mathrm{~cm}^{-1}$ ) are discussed. For this channel, a typical plot of the apodized noise equivalent spectral radiance (NESR) under flight conditions is shown in Fig. 2. In the spectral range between 820 and $970 \mathrm{~cm}^{-1}$, an optimal mean NESR of about $8 \times 10^{-9} \mathrm{~W} /\left(\mathrm{cm}^{2} \mathrm{srcm}^{-1}\right)$ is obtained, and at lower and higher wavenumbers, the NESR is still mainly around 1 to $2 \times 10^{-8} \mathrm{~W} /\left(\mathrm{cm}^{2} \mathrm{srcm}^{-1}\right)$.

The electronics module consists of a hierarchic transputer network with a PC-based computer as top system. Subsystems are the interferometer electronics, the line-of-sight (LOS) electronics and the housekeeping/auxiliary electronics. The system is designed for fully automatic operation during flight, but can be monitored and commanded via an Iridium satellite link (see http://www.iridium.com/default.aspx) during flight.

Since the measurements are performed at fixed tangent altitudes/elevation angles, an accurate line-of-sight (LOS) sta-
Table 1. Characteristics of MIPAS-STR in the current configuration.

\begin{tabular}{|c|c|}
\hline Telescope & \\
\hline Field of view (full cone) & $0.44^{\circ}$ \\
\hline Etendue & $2.6 \times 10^{-3} \mathrm{srcm}^{2}$ \\
\hline \multicolumn{2}{|l|}{ Interferometer } \\
\hline Effective optical path difference & $\pm 13.9 \mathrm{~cm}$ \\
\hline Scan time per interferogram & $\sim 9.5 \mathrm{~s}$ \\
\hline Sampling frequency & $48.8 \mathrm{kHz}$ \\
\hline Signal frequency & $2.1-6.1 \mathrm{kHz}$ \\
\hline Unapodized/apodized spectral resolution & $0.036 \mathrm{~cm}^{-1} / 0.058 \mathrm{~cm}^{-1}$ \\
\hline Detectors & $\mathrm{Si}: \mathrm{As}-\mathrm{BIB}$ \\
\hline \multicolumn{2}{|l|}{ NESR (single apodized spectrum, in-flight) } \\
\hline Channel $1\left(725-990 \mathrm{~cm}^{-1}\right)$ & $1 \times 10^{-8} \mathrm{~W} /\left(\mathrm{cm}^{2} \mathrm{srcm}^{-1}\right)$ \\
\hline Channel $2\left(1150-1360 \mathrm{~cm}^{-1}\right)$ & $8 \times 10^{-9} \mathrm{~W} /\left(\mathrm{cm}^{2} \mathrm{srcm}^{-1}\right)$ \\
\hline Channel $3\left(1560-1710 \mathrm{~cm}^{-1}\right)$ & $5 \times 10^{-9} \mathrm{~W} /\left(\mathrm{cm}^{2} \mathrm{srcm}^{-1}\right)$ \\
\hline Channel $4\left(1810-2100 \mathrm{~cm}^{-1}\right)$ & $5 \times 10^{-9} \mathrm{~W} /\left(\mathrm{cm}^{2} \mathrm{srcm}^{-1}\right)$ \\
\hline \multicolumn{2}{|l|}{ Pointing } \\
\hline Pitch/roll accuracy (AHRS) & $0.5 \operatorname{arcmin}(1 \sigma)$ \\
\hline Yaw accuracy (AHRS) & $0.3^{\circ}(1 \sigma)$ \\
\hline Estimated LOS-elevation accuracy & $0.78 \operatorname{arcmin}(1 \sigma)$ \\
\hline \multicolumn{2}{|l|}{ Dimensions } \\
\hline Optics module & $135 \times 75 \times 75 \mathrm{~cm}^{3}$ \\
\hline Electronics module & $50 \times 50 \times 50 \mathrm{~cm}^{3}$ \\
\hline Total mass & $\sim 200 \mathrm{~kg}$ \\
\hline Power consumption & $\sim 300 \mathrm{~W}(28 \mathrm{~V} \mathrm{DC})$ \\
\hline
\end{tabular}

bilization is required for compensating roll-variations of the aircraft. The development and verification of the LOS stabilization of MIPAS-STR is described by Keim (2002). The angle of the scan-mirror is measured by a 19-bit encoder and stabilized by a motor with reference to the attitude information provided by the Attitude and Heading Reference System (AHRS), which is part of the instrument. The AHRS is a Schuler-adapted, north-seeking, strapdown inertial navigation system with embedded GPS and a 10-state Kalmanfilter. The AHRS provides attitude angles at a data rate of $128 \mathrm{~Hz}$ and low latency, allowing for a near real-time LOS stabilization. After flight, the LOS data is refined by a dedicated post-processing procedure, compensating for drifts in the AHRS data.

Under flight conditions, a slight misalignment of the optical axis of the instrument with respect to the coordinate system of the AHRS can happen. The observed misalignment is mainly attributed to the exposure of the instruments housing to the large temperature difference between ground and stratosphere (in the order of $50 \mathrm{~K}$ ). For the compensation of this effect, the offset of the LOS with reference to the AHRS coordinate system is quantified by LOS retrievals (see Sect. 6). The retrieved LOS offset is found to be approximately stable under the relatively constant flight conditions in the stratosphere for individual flights. For each flight, one single LOS offset-parameter is determined and considered 


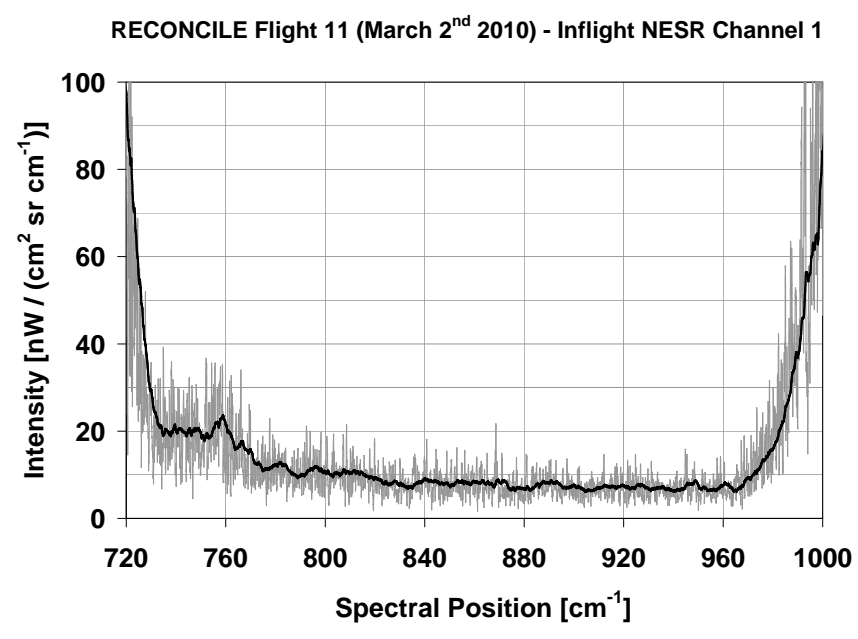

Fig. 2. Typical NESR of a single channel 1 spectrum under flight conditions (grey curve) and moving average (black curve).

for the subsequent retrievals of temperature and trace gases. Including the uncertainties inherent to the AHRS, the accuracy of the scan-mirror adjustment and the uncertainties resulting from the LOS retrieval, a total pointing accuracy of $0.78 \operatorname{arcmin}(1 \sigma)$ is estimated. This value corresponds to about $3 \%$ of the instrumental FOV or about $100 \mathrm{~m}$ at the lowest tangent altitude.

\section{Sampling}

The sampling programme of MIPAS-STR includes atmospheric measurements and calibration measurements. The atmospheric measurements with negative elevation angles, which are characterized by their tangent altitudes, allow for the retrieval of vertically resolved profiles of atmospheric temperature and trace gases below flight altitude. Measurements with positive elevation angles allow for the retrieval of the column amounts of these parameters above the flight path and also contain limited information on the vertical distributions directly above flight altitude. In Fig. 3, the sampling scheme of the standard limb sequence is illustrated. The atmospheric measurements are enclosed by blackbody and zenith view calibration measurements. Since the limb scans are aligned in a mirrored pattern, two full scans are shown. The standard sequence includes limb-viewing geometries with tangent altitudes between $5 \mathrm{~km}$ and flight altitude (vertical spacing mainly $1 \mathrm{~km}$ or $1.5 \mathrm{~km}$ ) and comprehensive upward sampling. For a typical flight altitude of $18 \mathrm{~km}$, one full limb scan including calibration measurements takes about $3.8 \mathrm{~min}$, corresponding to a flight path of approximately $45 \mathrm{~km}$. The horizontal distances of the tangent points from the aircraft position increase from about $33 \mathrm{~km}$ for the highest limb view (tangent altitude $17.9 \mathrm{~km}$, elevation angle of $-0.30^{\circ}$ ) to about $400 \mathrm{~km}$ for the lowest limb

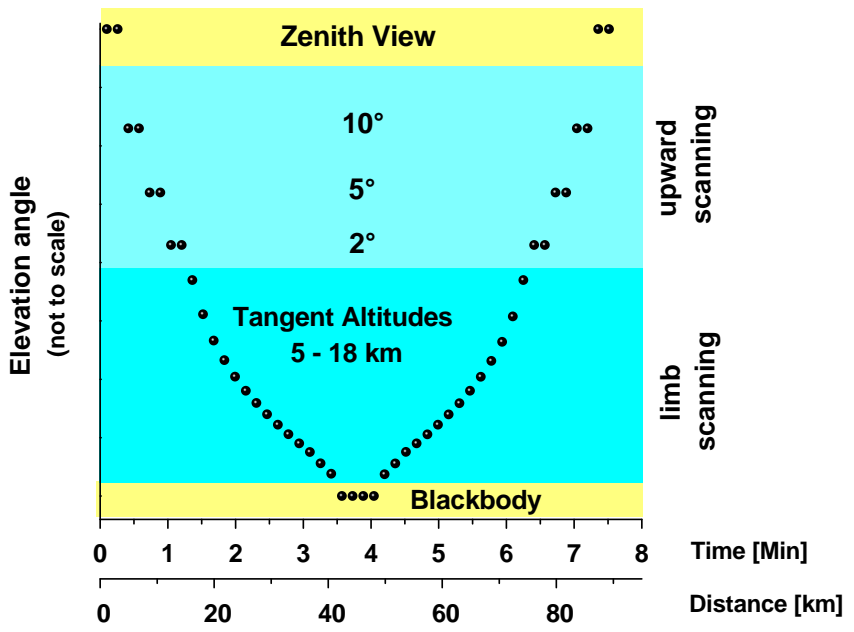

Fig. 3. Schematic representation of the standard sampling programme of MIPAS-STR, including atmospheric measurements (cyan) and calibration measurements (yellow). Two full limb scans are shown.

view (tangent altitude $5 \mathrm{~km}$, elevation angle of $-3.54^{\circ}$ ). The vertical FOV diameter at the tangent point increases for the respective geometries from about $0.3 \mathrm{~km}$ to $3.0 \mathrm{~km}$. Taking into account the vertical FOV angle of $0.44^{\circ}$, an oversampling with a factor of $2-3$ is obtained for the limb-viewing geometries.

In the presence of opaque tropospheric clouds or in case of the requirement for an increased horizontal sampling density at higher altitudes, a modified measurement scenario is applied. Tangent altitudes below $9 \mathrm{~km}$ are omitted and upwardscanning is performed less frequently, resulting in a total time of about $2.4 \mathrm{~min}$ for one full limb scan, corresponding to a horizontal sampling along flight track of approximately $25 \mathrm{~km}$.

\section{Spectral and radiometric calibration}

Aspects of the spectral and radiometric calibration procedure are, for instance, described in Höpfner et al. (2000) and Keim (2002) and are related to the procedure for the balloon-borne instrument MIPAS-B2 (Friedl-Vallon et al., 2004). Here, we give a summary of the full calibration cycle specific to MIPAS-STR (see Fig. 4).

For the determination of the detector nonlinearity, dedicated measurements are carried out during each flight, and the detector nonlinearity is considered to be approximately constant during an individual flight. For the subsequent steps phase correction, determination of instrumental offset, and radiometric calibration, an individual flight is separated into parts, where (i) the instrumental phase is sufficiently stable and (ii) the instrumental offset and (iii) the radiometric gain function show only approximately linear variations, since the 


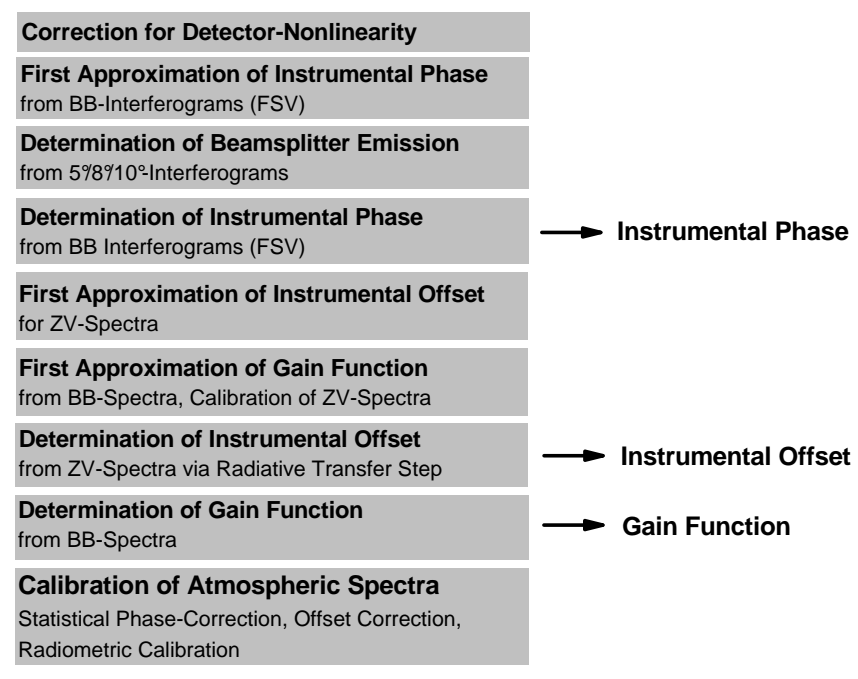

Fig. 4. Schematic representation of the full calibration cycle for MIPAS-STR measurements, including the determination of the detector non-linearity, instrumental phase, instrumental offset and radiometric gain function $(\mathrm{BB}=$ blackbody measurements, $\mathrm{ZV}=$ zenith view measurements, FSV = phase correction according to Forman, Steel and Vanasse, for details see text).

instrumental phase is averaged and the latter two quantities are fitted linearly in time for the atmospheric measurements. The latter three steps are carried out separately for each interferometer scan direction (forward/backward sweeps are carried out alternatingly), since the data-acquisition is slightly different.

\subsection{Detector nonlinearity correction}

The Si:As back-illuminated band impurity detectors show a significantly nonlinear response at increasing photon fluxes, resulting in a distortion of the interferograms and artefacts in the associated spectra.

The quantification of the detector nonlinearity for the balloon-borne instrument MIPAS-B2 is described in Kleinert (2006) and is carried out analogously for MIPAS-STR. The effects of nonlinearity are deduced and corrected from the higher order artefacts in blackbody spectra from interferograms without digital filtering data reduction as a function of the corresponding DC-level measured at the detector.

Depending on the availability of measurements of sufficient quality, between 15 and 30 interferograms are averaged typically for each interferometer scan direction and converted into spectra. The resulting two spectra are averaged again and from the final resulting spectrum the nonlinearity is quantified by the minimization of the observed artefacts. In the case of the channel 1 spectra of MIPAS-STR, the quadratic and cubic artefacts are clearly apparent and are minimized in the spectral interval from 30 to $280 \mathrm{~cm}^{-1}$ for the quadratic and in the interval from 2150 to $2900 \mathrm{~cm}^{-1}$ for the cubic artefacts. No signs of higher order artefacts are found. For RECONCILE flight 11 on 2 March 2010 discussed in this context, an $18 \%$ lower detector response is found for a typical blackbody spectrum compared to a zenith view spectrum.

\subsection{Phase correction of the interferograms}

Since different contributions of radiation arise from the atmosphere and the different optical components inside the instrument, complex spectra with a natural phase are resulting from the interferograms after the Fast-FourierTransformation (see Friedl-Vallon et al., 2004). Basically, atmospheric radiation and radiation resulting from the instruments optical components between the beamsplitter of the interferometer and the detector contribute to the real part of the spectrum, while the self-emission of the beamsplitter contributes mainly to the imaginary part. Due to the frequencydependent signal propagation in time in the optical dispersive elements and the electronic components, as well as sampling shifts relative to the interferogram peak, phase errors result. A dedicated approach suitable for the phase correction for MIPAS instruments has been established by Trieschmann et al. (1999) and Kleinert and Trieschmann (2007). A characteristic instrumental phase is determined using blackbody calibration measurements and is then used as initial phase for a statistical phase correction of the atmospheric interferograms (see Fig. 4).

Following this approach, in the first step an initial mean instrumental phase is determined from the blackbody measurements of the corresponding flight section according to Forman et al. (1966). The resulting preliminary instrumental phase is then applied for the phase correction of the $5^{\circ} / 8^{\circ} / 10^{\circ}$-measurements (elevation angles depending on the sampling sequence), which show only weak atmospheric signatures and allow for the extraction of the beamsplitter emission in the imaginary part. The resulting imaginary spectra, which are characterized by the beamsplitter emission pattern, are then averaged and smoothed in order to reduce the noise level without affecting the broad signatures resulting from the beamsplitter emission. In the next step, the improved final instrumental mean phase is determined from the blackbody measurements according to Forman et al. (1966), taking into account the beamsplitter emission derived in the previous step. The resulting instrumental phase serves as starting point for the statistical phase correction of the zenith view and atmospheric interferograms. In the statistical phase correction (Trieschmann et al., 1999), (i) the correlations between the real and imaginary parts of the spectra are minimized, since these parts are theoretically absolutely independent from each other and (ii) the variances in the imaginary parts are minimized, since no sharp line features are expected here.

After the phase correction, the real parts of the atmospheric spectra, which contain the atmospheric signatures of 


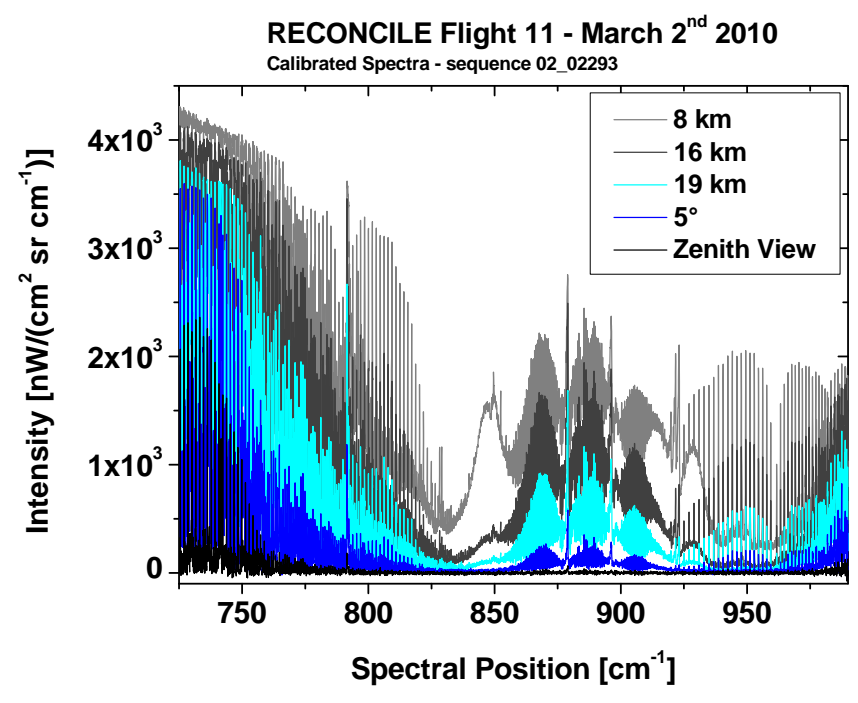

Fig. 5. Examples for single calibrated spectra from RECONICLE flight 11 on 2 March 2010. In the box in the upper right side, the corresponding tangent altitudes/elevation angles of the spectra are listed $\left(0^{\circ}\right.$ elevation corresponds to horizontal view).

interest, and the zenith view spectra, allowing for the extraction of the instrumental background radiation, are further processed in order to obtain final calibrated atmospheric spectra.

\subsection{Determination of instrumental offset and radiometric calibration}

The instrumental offset in the real parts of the spectra is determined from the zenith view spectra following the iterative scheme developed by Höpfner et al. (2000). Since the zenith view spectra are obtained from observations towards cold space, only weak atmospheric rest-signatures are apparent, and further remaining contributions represent the instrumental background radiation. In order to reproduce the instrumental background with maximal accuracy, the remaining atmospheric rest-signatures are removed by a radiative transfer step with KOPRA/KOPRAFIT (see Sect. 6.1). For this purpose, a preliminary calibration is applied to the zenith view spectra, including a line fitting step without radiative transfer and a first determination of the gain function from the blackbody measurements. In the radiative transfer step, the remaining weak signatures of $\mathrm{CO}_{2}, \mathrm{O}_{3}, \mathrm{HNO}_{3}$ and $\mathrm{H}_{2} \mathrm{O}$ are retrieved and then subtracted from the measured zenith view spectra, resulting in the desired instrumental offset spectra.

With the knowledge of the instrumental offset, the final radiative gain function is determined from the blackbody measurements according to Eq. (1):

$c(v)=\frac{\mathrm{BB}(v)-U(v)}{B(v, T) \cdot e(v)}$,

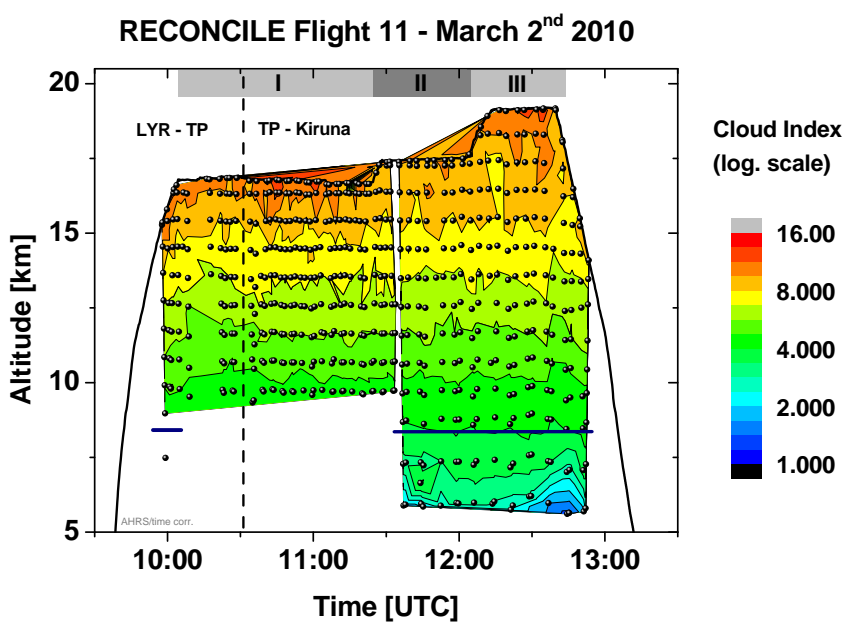

Fig. 6. Vertical distribution of tangent points (points) and interpolated cloud index (contour). Flight altitude of the Geophysica (solid black line) along flight track from Longyearbyen (LYR) via the turning point (TP, dashed black line) to Kiruna. The horizontal blue lines represent the approximate threshold for cloud index 4 .

whereas $v$ stands for the spectral position, $c(v)$ for the radiative gain function, $\mathrm{BB}(v)$ for the blackbody spectrum, $U(v)$ for the instrumental offset spectrum, $B(\nu, T)$ for the Planck function of the temperature $T$ and $e(v)$ for the emissivity of the blackbody. With the knowledge of the precise instrumental offset and the radiative gain function, the atmospheric spectra are calibrated according to the two-point calibration approach:

$S(v)=\frac{A(v)-U(v)}{c(v)}$,

whereas $S(v)$ represents the calibrated atmospheric spectrum and $A(v)$ the atmospheric raw spectrum.

In Fig. 5, examples of calibrated atmospheric spectra are shown together with a calibrated zenith view spectrum. The zenith view spectrum and the spectra with high elevation angles indicate a flat baseline and show no signs of any significant radiometric offset. For lower elevation angles, the spectral baseline is superimposed by overlapping signatures of trace gases and significant continuum-like contributions, which have to be considered in the retrievals.

\section{Flight description}

RECONCILE flight 11 was carried out on 2 March 2010, starting from Longyearbyen (Spitsbergen) at 09:35 UTC and landing in Kiruna (Sweden) at 13:35 UTC. The flight was designated as the second part of a double-flight from Kiruna to Longyearbyen and return, and was situated in the late phase of the polar vortex 2009/2010 after a vortex split in February. During the time of the flight, a compact vortex remnant was passing Spitsbergen coming from 
RECONCILE Flight 11 - March $2^{\text {nd }} 2010$

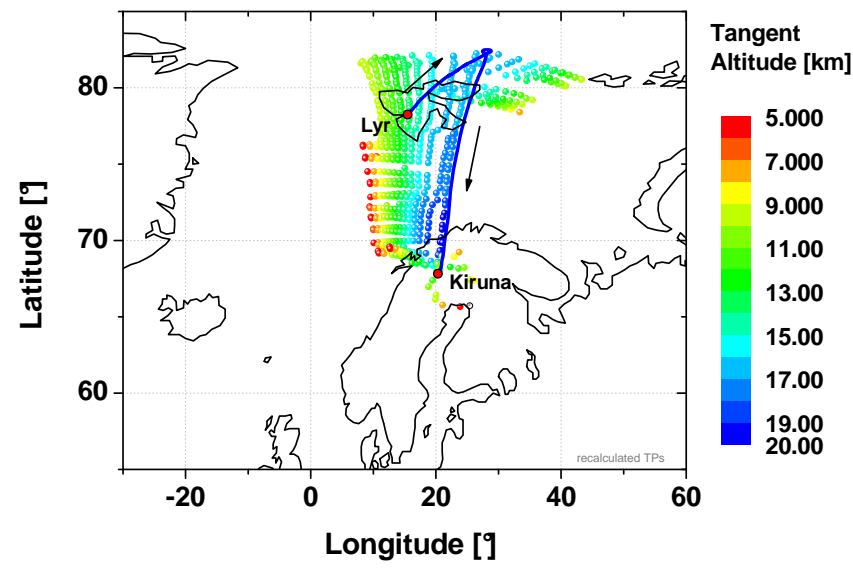

Fig. 7. Flight track of the Geophyscia for RECONCILE flight 11 (solid blue line) and distribution of tangent points of the MIPASSTR observations, colour-coded with reference to the tangent altitude.

Canada, allowing for the sampling of aged vortex air and vortex filaments.

The vertical distribution of the tangent points of the MIPAS-STR observations along the flight track is shown in Fig. 6 (upward-viewing geometries not indicated) together with the cloud index. All shown tangent altitudes correspond to final measurement geometries after LOS-correction from retrieval. During the first part of the flight, the modified limb sequence omitting nominal sampling below $9 \mathrm{~km}$ was carried out, in order to achieve a high horizontal sampling density in the vortex air. Beginning from 11:35 UTC, the standard sequence with nominal sampling down to $5 \mathrm{~km}$ was applied, in order to increase the vertical coverage.

According to Spang et al. (2004), the cloud index is derived from the spectra as the colour ratio between the microwindows 788.20 to $796.25 \mathrm{~cm}^{-1}$ and 832.30 to $834.40 \mathrm{~cm}^{-1}$, and is indicated colour-coded in Fig. 6. While spectra with cloud indices higher than 4 are assigned as cloud-free, cloud indices between 1 and 4 indicate partly cloud-affected spectra and values close to 1 indicate clearly cloud-affected spectra. Since the cloud index described in Spang et al. (2004) is derived for MIPAS and CRISTA spectra from satellite orbits and is defined for stratospheric altitudes, the threshold for cloud-affected spectra is likely to be slightly different for MIPAS-STR, especially at tropospheric altitudes. In fact, further studies (not published) indicate that MIPAS-STR spectra with cloud index 3 are still retrievable without constraints. However, in this context the conservative cloud index threshold of 4 is applied in order to avoid any significant cloud-effects in the retrievals. While in Fig. 6 clearly cloud-affected spectra (cloud-index close to 1) are only visible towards the very end of the flight at the lowest tangent points, spectra assigned as partially cloud affected (cloud indices between 1 and 4) are found for most

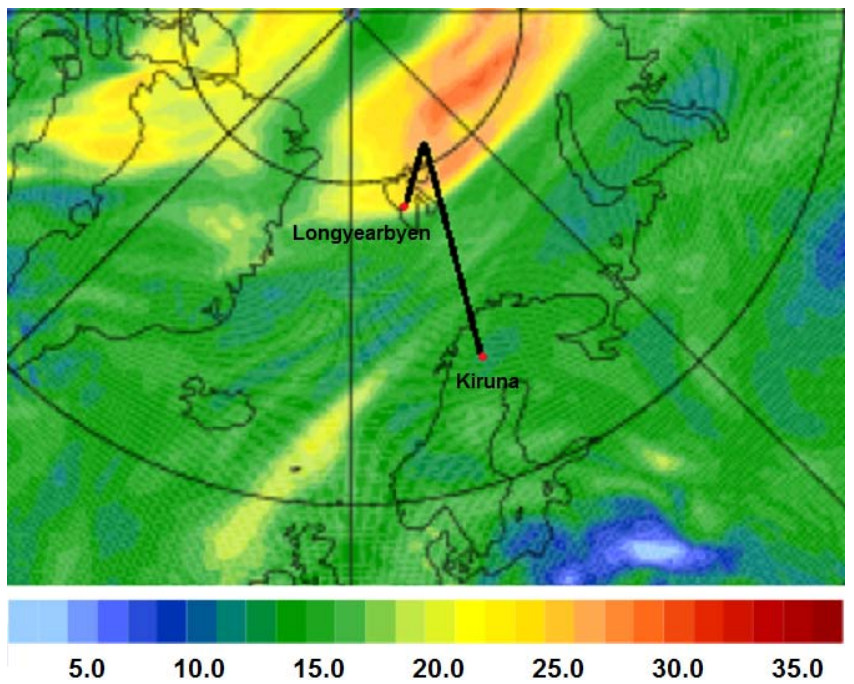

Fig. 8. Meteorological situation during RECONCILE Flight 11. The colour-coding of the map shows the scaled potential vorticity at the $450 \mathrm{~K}$-level (approximately $17 \mathrm{~km}$ altitude). A late vortex remnant, coming from Canada and reaching from Spitsbergen to Siberia, can be identified clearly. The flight path of the Geophysica is indicated schematically.

geometries with tangent points below $8.5 \mathrm{~km}$, and the corresponding spectra are omitted in the retrievals.

In Fig. 7, the flight track is shown together with the horizontal distribution of the tangent points. Since MIPAS-STR is pointing towards the right hand side of the Geophysica, the area in the south-east of the flight track was sampled during the first flight section before the turning point. After the turn at approximately $82^{\circ} \mathrm{N}$, the instrument was sampling the area west of the flight track.

In Fig. 8, the meteorological context during the discussed RECONCILE Flight 11 is illustrated based on ECMWF operational analyses. The map of the scaled potential vorticity (see Dörnbrack et al., 2012) at the $450 \mathrm{~K}$ level (approximately coinciding with the flight altitude of the Geophysica between 10:00 UTC and 12:00 UTC) shows the late vortex remnant coming from Canada, passing Spitsbergen with the lower edge from the north-west. The map reflects the meteorological situation at 12:00 UTC, coinciding well with the time of the flight. As can be seen from Figs. 7 and 8, the Geophysica ascended from Longyearbyen at Spitsbergen into the Canadian vortex remnant. After the turn at approximately 10:30 UTC towards the southward flight leg, different airmasses covering vortex air and extra-vortex air were passed subsequently. An extended vortex filament can be identified in Fig. 8 at the Atlantic western coast of Scandinavia, which is covered partially by the tangent points of MIPAS-STR during the southern flight section. It has to be noted that vortex air and extra-vortex air can contribute to individual spectra of MIPAS-STR at the same time as a consequence of the horizontally extended viewing geometries. 


\section{Retrieval}

\subsection{Retrieval method}

The retrieval of the atmospheric parameters is carried out using the forward model Karlsruhe Optimized and Precise Radiative Transfer Algorithm (KOPRA) (Stiller et al., 2000) and the inversion module KOPRAFIT (Höpfner et al., 2001).

KOPRA is a dedicated fast line-by-line code for the modelling of spectra from high-resolution spectrometers. It allows for including both limb mode and upward scanning geometries together for the same retrieved profile. Various atmospheric aspects of radiative transfer and instrumental aspects are supported, including non-spherical ray-tracing, refraction, FOV and ILS. Spectral lines of target gases are modelled using the Voigt profile, and for species with unresolved signatures available absorption cross-section data is applied.

Utilizing the analytical derivatives provided by KOPRA, the inversion algorithm KOPRAFIT allows for the fitting of the full set of observations of one scan in many microwindows simultaneously. For the inversion of atmospheric parameter profiles in this context, Gauss-Newton iteration subjected to Tikhonov-Phillips regularization (Tikhonov, 1963; Phillips, 2003) is applied:

$$
\begin{aligned}
\boldsymbol{x}_{i+1}=\boldsymbol{x}_{i}+\left(\mathbf{K}_{i}^{\mathrm{T}} \mathbf{S}_{y}^{-1} \mathbf{K}_{i}+\gamma \mathbf{L}^{\mathrm{T}} \mathbf{L}\right)^{-1} \\
\quad\left[\mathbf{K}_{i}^{\mathrm{T}} \mathbf{S}_{y}^{-1}\left(\boldsymbol{y}-\boldsymbol{f}\left(\boldsymbol{x}_{i}\right)\right)+\gamma \mathbf{L}^{\mathrm{T}} \mathbf{L}\left(\boldsymbol{x}_{\mathrm{a}}-\boldsymbol{x}_{i}\right)\right] .
\end{aligned}
$$

Here, $i$ represents the iteration-index, $\boldsymbol{x}_{i}$ stands for the vector with the retrieval quantities, $\boldsymbol{x}_{\mathrm{a}}$ for the a priori-profile, $\boldsymbol{y}$ represents the vector with the measured radiances, $\mathbf{K}_{i}$ is the spectral derivatives matrix (Jacobian) with respect to the retrieved quantities, $\mathbf{S}_{y}$ the variance-covariance matrix of the measurements and $\boldsymbol{f}$ the forward-model. The TikhonovPhillips regularization is realized by the first-order regularization operator $\mathbf{L}$ and the regularization strength is adjusted by the regularization parameter $\gamma$. An advantage of the applied Tikhonov-Phillips smoothing constraint is the fact that systematic biases with respect to absolute values of the target parameters are avoided rather than using a climatological constraint (e.g. Steck, 2002). The regularization parameters for the target parameters temperature, trace gases and background-continuum are optimised individually, avoiding oscillations in the results and considering the residuals between the measured and retrieved spectra.

\subsection{Characteristics of continuum-affected spectra from the UTLS}

The altitude range resolved by the measurements of MIPASSTR is situated mainly in the UTLS region between 5 and $20 \mathrm{~km}$. In this range, effects referred in this context as "continuum-like contributions" are important in the spectra and have to be taken into account in the retrievals. Typical greybody-like continua in the spectra are resulting from clouds, and significantly cloud-affected spectra are filtered according to the cloud index from Spang et al. (2004) described in Sect. 5. Possible reasons for further continuumlike effects (under nominal cloud-free conditions) in the spectra on the scale of retrieval microwindows are

- Low concentrations of aerosol accumulated along the line of sight.

- Broad spectral signatures of trace gases.

- Spectral line-shape related effects.

The first aspect includes effects from all types of aerosol. Ubiquitary, stratospheric, sulphur-containing aerosols are associated with the global Junge-Layer (Junge et al., 1961) and can result from volcanic eruptions (e.g. Arnold and Bührke, 1983), anthropogenic influences and biogeochemical sources (e.g. Hofmann, 1990; Andreae and Crutzen, 1997). Different types of particles also can result from or nucleate on meteoritic material (e.g. Curtius et al., 2005). Polar Stratospheric Cloud (PSC) particles are formed during winter at high latitudes (e.g. Peter, 1997) and significantly affect mid-infrared limb-emission spectra (e.g. Höpfner et al., 2006). Also cirrus cloud particles affect mid-infrared limb-emission spectra (e.g. Spang et al., 2002) and the corresponding effects can be taken into account by dedicated radiative transfer models (Ewen et al., 2005). Further types of stratospheric aerosolparticles were measured by in-situ instruments, whereas the sources for some particle types remain unclear (e.g. Murphy et al., 1998). So in summary, there can be different aerosolfamilies present in the UTLS region under nominal cloudfree conditions, which are capable of affecting the MIPASSTR observations.

Regarding the second point, continuum-like signatures on the scales of retrieval microwindows (typically several $\mathrm{cm}^{-1}$ ) also result from large molecules with characteristic broad signatures in the spectra, which are omitted or inaccurately covered by radiative transfer modelling. Here, the availabilities of suitable climatological profiles, as well as accurate spectral line-data and cross-sections represent limiting factors. Candidates are, e.g. the broad family of the halocarbons (e.g. Fabian and Borchers, 1981) and probably also aliphatic and aromatic hydrocarbons. Although many species alone give rise to only weak signatures below or close to the NESR of MIPAS-STR, the net-effect accumulated by many different species can significantly affect the observations.

The third aspect results from the significantly increasing pressure broadening of spectral lines observed with decreasing altitude in the UTLS region. As a consequence of the increasing Lorentzian character of the spectral lines and the overall increase of many spectral signatures towards lower observation geometries, the overlap of adjacent spectral signatures increases. For the consideration of the effects of overlapping line wings, spectral signatures in an extended wavenumber-range of $25 \mathrm{~cm}^{-1}$ around the selected 
microwindows are taken into account in the retrievals discussed here. Influences of the extreme line-wings of very strong distant $\mathrm{CO}_{2}$-lines and the non-Lorentzian behaviour of $\mathrm{H}_{2} \mathrm{O}$-lines are considered by dedicated continuum models included in KOPRA (Stiller et al., 2000). However, remaining broad continuum-like spectral signatures resulting from the incomplete modelling of overlapping spectral signatures cannot be ruled out. Virtually continuum-like contributions might also result from imperfect spectral modelling of adjacent spectral signatures, using the Voigt model, since it does not always allow for a sufficient reconstruction of the observed spectral line shapes (e.g. Ciurylo, 1998; Boone et al., 2007; Schneider et al., 2011). However, we clarify that the retrieval of background-continuum does not correct imperfect spectral modelling of line shapes, but rather might partially compensate net-effects resulting from incorrectly modelled line-wings of adjacent spectral signatures.

Furthermore, we mention that also stray-light effects resulting from impurities on the instruments scanning mirror might result in spectral contributions possibly interpreted as continuum-like contributions.

So in summary, different superimposed so-called continuum-like contributions play a role in UTLS midinfrared limb-emission FTIR spectra, with increased importance towards lower altitudes. The sources for continuum-like effects are variable with altitude and for different spectral regions, and consequently a clear and quantitative separation is complicated. Therefore, the neteffect of continuum-like contributions in the measurements analysed here is quantified by the retrieval of a wavenumberindependent background continuum, allowing for a reliable reconstruction of the main target parameters. Of course it has to be considered that the main retrieval target has to show a signature sufficiently differing from continuum-like behaviour in the spectral microwindow(s) chosen for the retrieval.

In Fig. 9a, a measured spectrum with a tangent altitude of $12.5 \mathrm{~km}$ is shown, where significant continuum-like effects are apparent. Also shown are the corresponding retrieved spectra with and without continuum-retrieval. The retrieval without consideration of background continuum results in poor agreement between measurement and retrieval result, while the retrieved spectrum with simultaneous reconstruction of background continuum reproduces the measured spectrum well. Remaining differences in the residual exceeding the NESR-level are attributed to signatures of weakly interfering trace gases, which are not identified or incorrectly modelled using climatological profiles. In Fig. 9b, the retrieved continuum profile from the corresponding full limb scan is shown for this microwindow and retrieval.

\subsection{Retrieval approach}

The full retrieval chain applied for the subsequent reconstruction of the LOS offset, temperature profiles and trace
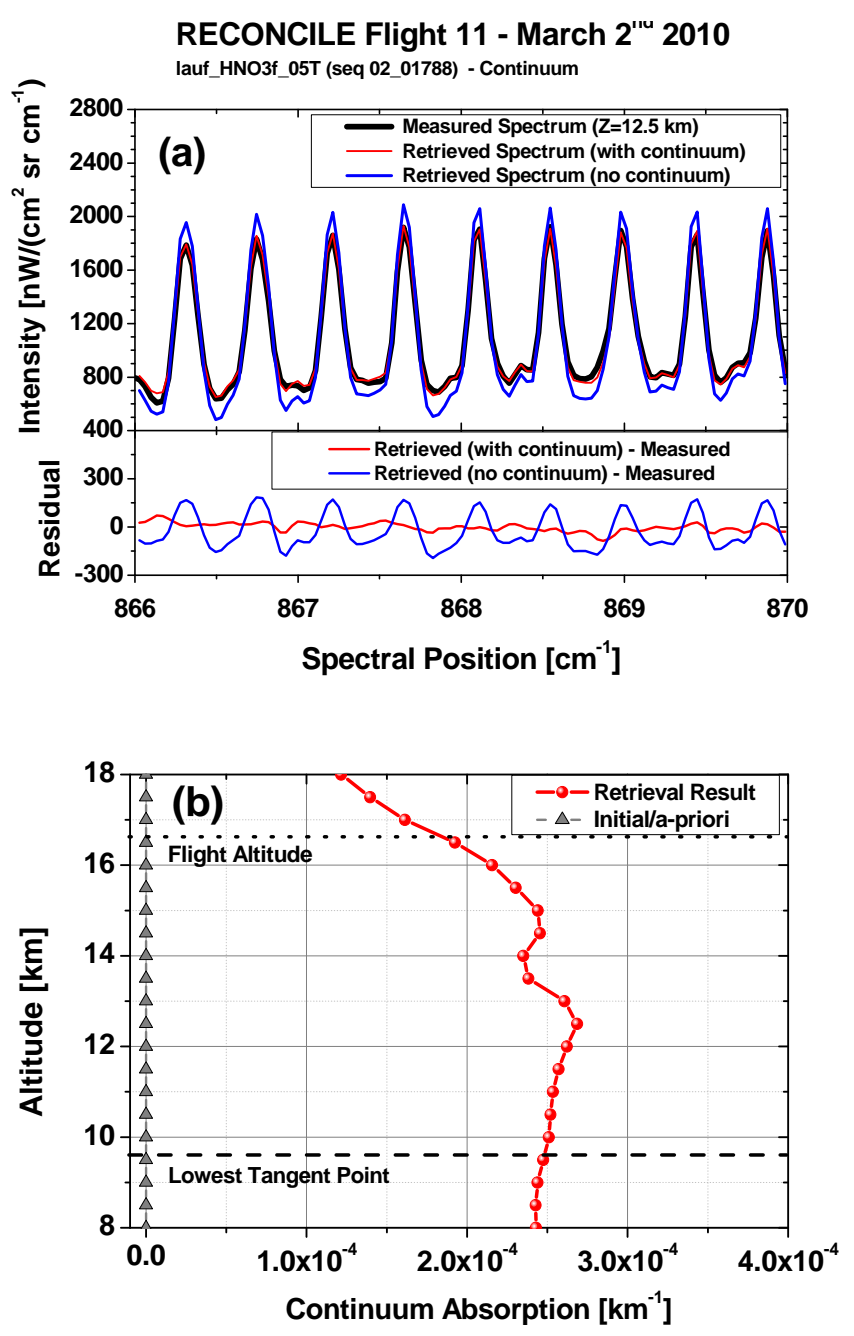

Fig. 9. (a) Measured spectrum (black) and retrieved spectra with (red) and without (blue) background continuum retrieval for a flight altitude of $16.7 \mathrm{~km}$ and a tangent altitude of $12.5 \mathrm{~km}$ for the $\mathrm{HNO}_{3}$ retrieval and residuals. (b) Associated retrieval result for continuum absorption of the corresponding full limb sequence.

gas profiles is summarized in Fig. 10. As pointed out in Sect. 2, only a constant absolute offset between the reference system of the AHRS and the instruments LOS has to be quantified by retrievals. Therefore, the first step in the applied retrieval chain is the determination of the LOS offset using strong isolated $\mathrm{CO}_{2}$-lines in the spectra. For each sequence of a flight, an individual LOS offset parameter is retrieved, and the average of all retrieved LOS offset parameters is used as LOS correction. Thereby, the following assumptions are made: (i) The temperature and pressure profiles used for the LOS retrieval, which are interpolated from the ECMWF T106 grid-point analysis, are correct on average during a flight. (ii) The applied $\mathrm{CO}_{2}$-profile and spectral line data for the modelling of the $\mathrm{CO}_{2}$-lines are also sufficiently accurate. (iii) Further significant errors, e.g. resulting 


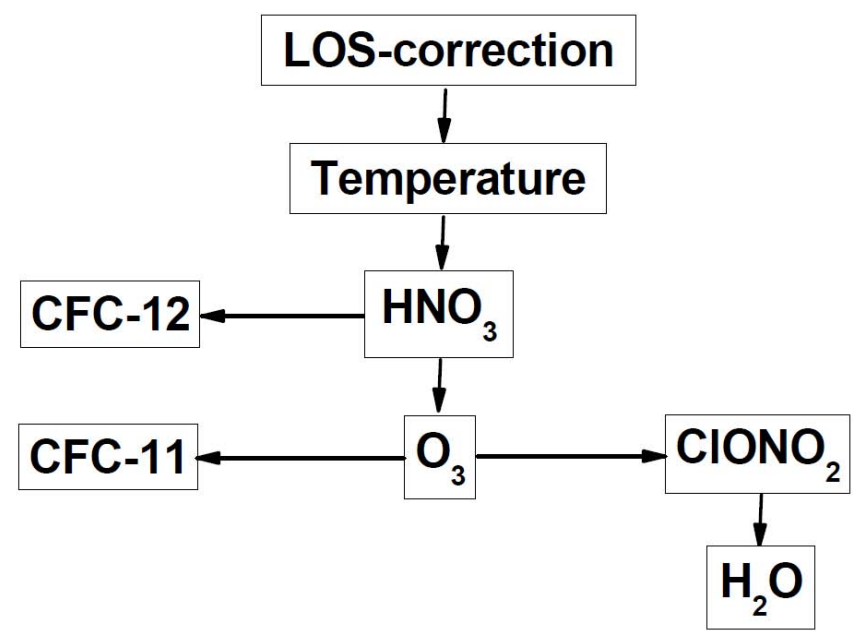

Fig. 10. Schematic representation of the subsequent retrievals of LOS correction, temperature and the discussed trace gases from MIPAS-STR channel 1 spectra. For each target parameter, all other parameters prior in the scheme are determined previously.

from uncertainties in the relative LOS-information (due to the limited accuracy of the AHRS data) or radiometric calibration, show no significant systematic behaviour during a flight. (iv) The LOS offset is approximately constant during a flight.

In the next step indicated in Fig. 10, temperature is retrieved for all limb sequences, using the same spectral signatures with strong isolated $\mathrm{CO}_{2}$-lines as for the LOS retrieval. The lines fulfil the requirements or advantages for a temperature retrieval of (i) sufficiently strong intensity, (ii) being clearly separable from other spectral signatures, (iii) different opacity and (iv) different temperature-dependence. It has to be noted that the absolute situation (i.e. position in altitude) of the retrieved temperature profiles is clearly affected by the previously determined LOS correction, relying on interpolated temperature profiles from ECMWF and making use of the same spectral signatures. But on the other hand, only one single constant LOS correction is applied for all limb sequences of a flight, and no vertical information within the profile is retrieved from the $\mathrm{CO}_{2}$-signatures in the determination of the LOS correction.

According to Fig. 10, the different trace gases are retrieved subsequently in the following steps. First, target gases with dominant signatures and low spectral interference with other species are retrieved; then, with the optimised knowledge of the profiles of these gases, the corresponding spectral signatures are modelled for the retrieval of further trace gases with signatures influenced by the previously retrieved gases. Using previously fitted fixed profiles of significantly interfering species rather than climatological profiles allows for an improved modelling, which is especially important when spectra of air-masses from different origins (e.g. vortex and extravortex air) are retrieved within single limb scans. If possi- ble, the simultaneous reconstruction of interfering gases is avoided, since the total number of fit-parameters is increased and the simultaneous reconstructed parameters can potentially compensate each other to a certain extent, especially in the context of the continuum-retrieval.

The vertical profile of $\mathrm{H}_{2} \mathrm{O}$ shows a strong tropospheric gradient in contrast to the only weakly varying stratospheric mixing ratios. Therefore, logarithmic inversion is applied, resulting in more reasonable results in this particular case than by applying linear inversion as for the other retrievals. It has to be mentioned that for the other retrieval target parameters microwindows with no significant spectral interference with $\mathrm{H}_{2} \mathrm{O}$ signatures are used, since several signatures of $\mathrm{H}_{2} \mathrm{O}$ strongly increase towards tropospheric altitudes and complicate retrievals of other parameters.

\subsection{Retrieval setup}

For the retrievals, a grid between $0-100 \mathrm{~km}$ with a spacing of $0.5 \mathrm{~km}$ below $30 \mathrm{~km}$ and increasing spacing above is applied. Regularization is needed, since the applied retrieval grid in the vertical range of interest (spacing of $0.5 \mathrm{~km}$ ) is finer than measurement grid (mainly spacing of $1 \mathrm{~km}$ ) and the vertical FOV of the instrument. However, considering the complex combination of (i) the increase of the vertical FOV diameter with decreasing tangent altitude, (ii) oversampling, (iii) the non-strictly conducted $1 \mathrm{~km}$-sampling grid in the specific limb programs, (iv) the corrected LOS variations quantified in the AHRS-post-processing altering the sampling grid, and (v) the effects of the inhomogeneous vertical FOV, the combination of a $0.5 \mathrm{~km}$ retrieval grid with an optimized regularization is found to allow for a comprehensive use of the vertical information included in the spectra.

The utilized spectral microwindows for the retrievals and the most prominent target signatures are summarized in Table 2. Spectral line parameters and cross-sections for the retrievals are taken from the MIPAS database (Flaud et al., 2002, 2006). Only for $\mathrm{ClONO}_{2}$, cross-section data is taken from a study of Wagner and Birk (2003). Depending on the availability of suitable microwindows with sufficiently strong target signatures and low spectral interference with other gases, each 1 or 2 microwindows are selected for the individual retrievals, allowing for manageable and efficient optimization of the regularization parameters and fast computation. Retrieval parameters are (i) the target quantity (LOS offset/temperature/trace gases), (ii) wavenumber-independent background continuum for each microwindow and (iii) spectral shift of the microwindows. From the cases discussed here, only in the retrieval of the LOS correction and in the temperature retrieval $\mathrm{O}_{3}$ is reconstructed as an additional parameter. However, the corresponding signatures are clearly separable from the target-signatures of $\mathrm{CO}_{2}$, allowing for a reliable reconstruction of the main target parameter (the results for $\mathrm{O}_{3}$ with comparably low accuracy and vertical resolution are discarded subsequently). Regularization is applied 
Table 2. Microwindows and target signatures selected for the retrievals. Spectral positions of the most prominent target signatures are listed. For cross-section gases, the spectral signatures are relevant in the whole range of the microwindow.

\begin{tabular}{|c|c|c|c|}
\hline Retrieval target & $\begin{array}{l}\text { Microwindow } \\
{\left[\mathrm{cm}^{-1}\right]}\end{array}$ & Target signature & $\begin{array}{l}\text { Spectral position } \\
{\left[\mathrm{cm}^{-1}\right]}\end{array}$ \\
\hline $\begin{array}{l}\text { LOS/temperature } \\
\left(\mathrm{CO}_{2} \text { signatures }\right)\end{array}$ & $\begin{array}{l}810.1-813.1 \\
955.6-958.5\end{array}$ & $\begin{array}{l}\text { R24e }(11101 \leftarrow 10002) \\
\text { R26e }(11101 \leftarrow 10002) \\
\text { P6e }(00011 \leftarrow 10001) \\
\mathrm{P} 4 \mathrm{e}(00011 \leftarrow 10001)\end{array}$ & $\begin{array}{l}810.93 \\
812.48 \\
956.19 \\
957.80\end{array}$ \\
\hline $\mathrm{HNO}_{3}$ & $866.0-870.0$ & $v 5 / 2 \nu 9$ bands & $\begin{array}{l}9 \text { resolved } \\
\text { net peaks ( } v 5 \text {-band) }\end{array}$ \\
\hline $\mathrm{O}_{3}$ & $787.0-788.0$ & v2-band & $\begin{array}{l}780.80 \\
781.13 \\
781.18 \\
781.52 \\
787.13 \\
787.46 \\
787.86\end{array}$ \\
\hline CFC-11 & $842.5-848.0$ & v4-band & cross-section \\
\hline CFC-12 & $\begin{array}{l}918.9-920.6 \\
921.0-922.8\end{array}$ & $\begin{array}{l}\text { v6-band } \\
v 6 \text {-band }\end{array}$ & $\begin{array}{l}\text { cross-section } \\
\text { cross-section }\end{array}$ \\
\hline $\mathrm{ClONO}_{2}$ & $\begin{array}{l}779.8-780.5 \\
805.1-805.5\end{array}$ & $\begin{array}{l}\text { v4-band } \\
\text { v3-band }\end{array}$ & $\begin{array}{l}\text { cross-section } \\
\text { cross-section }\end{array}$ \\
\hline $\mathrm{H}_{2} \mathrm{O}$ & $795.7-796.1$ & pure rotation & 795.89 \\
\hline
\end{tabular}

to the retrieval parameters temperature, trace gases and background continuum and the regularization parameters are kept constant for all limb sequences of a flight.

Pressure profiles for all retrievals and temperature profiles for the LOS-retrieval are interpolated from the ECMWF analysis at T106 resolution.

In all discussed retrievals, initial guess and a priori profiles are identical for an individual retrieval parameter. For the temperature retrievals, the interpolated profiles from ECMWF are used as initial guess/a priori profiles. For the trace gas retrievals, the initial guess/a priori profiles are taken from the Polar Winter Profiles for MIPAS V3.1 of Remedios et al. (2007), slightly modified for the conditions of the Polar Winter 2009/2010 (i.e. $\mathrm{CO}_{2}$ mixing ratios updated). For the reconstruction of background continuum, the initial guess/a priori is set to zero, starting the retrieval with the assumption of no continuum. Signatures of weakly interfering trace gases, which are not retrieved, are considered by their climatological profiles from Remedios et al. (2007).

\subsection{Retrieval result characterization}

The retrieval results are characterized by the following quantifiers: (i) estimated error, (ii) vertical resolution and (iii) degrees of freedom. Different error-sources inherent to the measurement technique and the retrieval method are divided into two groups. The dominating errors, assigned as "primary errors", result from

- uncertainties in the spectroscopic line data,

- uncertainties of the applied temperature profiles,

- uncertainties of the LOS-information,

- radiometric calibration errors,

- spectral noise.

Further errors sources referred to "secondary errors" are

- uncertainties in the applied pressure profiles,

- uncertainties resulting from horizontal inhomogeneities in the atmosphere,

- uncertainties/incompleteness of the previously retrieved/climatological trace gas profiles used for forward-modelling of interfering species,

- uncertainties of trace gas profiles above the flight path,

- errors due to non-local thermodynamic equilibrium at high altitudes,

- line-mixing of spectral signatures,

- errors in the characterization of the FOV, 
- deviations of the real ILS from the theoretical model,

- errors resulting from the limited knowledge of the aircraft altitude (GPS),

- errors resulting from stray-light in the instrument,

- further errors resulting from the electronic data acquisition chain.

The primary errors are considered in the error estimation, while the secondary errors are expected to be of minor importance in most cases, and are not included in the errorbudget. Uncertainties of the interpolated pressure profiles are expected to have low impact as a consequence of the high quality of the ECMWF data. Errors due to the nonconsideration of horizontal gradients of atmospheric parameters along the line-of-sight are expected to be less important compared to the balloon-borne and satellite-borne MIPAS instruments as a consequence of shorter light paths through the atmosphere. However, in the presence of strong horizontal gradients and contrasts of trace gas mixing ratios, significant uncertainties in the retrievals cannot be excluded. On the other hand, to a limited extent, the effects of horizontal gradients in the retrievals can also be interpreted as a characteristic of the sampled air-mass as a whole rather than an error. Further uncertainties can arise from horizontal inhomogeneities along the flight track, since spectral contributions from a certain altitude regime can change within the recording of a limb sequence, but significantly affect different measurement geometries of the same sequence.

Errors resulting from potentially inaccurate modelling of interfering signatures using retrieved and climatological profiles are expected to be of minor importance, since spectral microwindows with dominating signatures of the target gas and weak signatures from known interfering gases are utilized. Furthermore, errors in the modelling of broad and weakly structured interfering signatures are at least partially compensated by the retrieval of background-continuum. However, we remark that at tropospheric altitudes the mixing ratios of several species strongly increase and show high variability so that the retrieval error due to interfering species can become important here.

The limited knowledge of the profiles of atmospheric constituents above the flight path represents a relevant error source in principle, especially in the case of trace gases where the maximum of the profile is located above the flight altitude. However, this uncertainty is reduced by considering the upward-viewing geometries included in the limb sequences, allowing for the reconstruction of column information above the flight path and to a limited extent also vertical information. The low impact of variable ozone above the flight path on the vertically resolved region of the retrieval for this gas below and around flight altitude (which is considered in the following sections) is shown in Fig. 11. Forward calculations were carried out based on two ozone profiles with

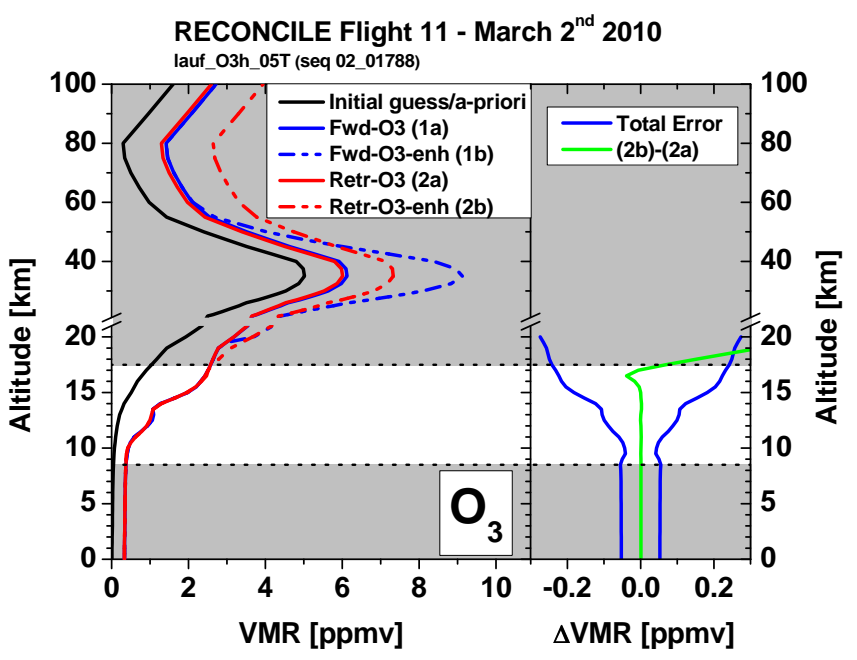

Fig. 11. Retrieval simulations for different ozone maxima above the flight path (flight altitude: $16.7 \mathrm{~km}$ ). Left panel: Profile Fwd-O3 (1a), derived from nominal processing of scan 02_01788, and FwdO3-enh (1b) with enhanced ozone above flight path were applied for forward calculations based on the viewing geometries, measurement noise and atmospheric conditions of scan 02_01788. The resulting forward spectra were retrieved again, yielding profiles RetrO3 (2a) and Retr-O3-enh (2b). Right panel: Comparison of total error of nominal retrieval result and difference between $(2 b)$ and (2a). Regions with vertical resolution of the nominal retrieval result worse than $5 \mathrm{~km}$ shaded in grey.

identical mixing ratios below $20 \mathrm{~km}$ and significantly different mixing ratios above. The corresponding forward spectra were retrieved using the same indicated initial guess and a priori profile, resulting in nearly identical profiles in the vertically resolved region of this limb scan between 8.5 and $17.5 \mathrm{~km}$.

Errors resulting from non-local thermodynamic equilibrium are expected to be practically insignificant for the measurement geometries of MIPAS-STR, since these effects are mainly important high above the flight altitude (e.g. Manuilova et al., 1998; von Clarmann, 2003). Possible errors resulting from line-mixing (e.g. Funke et al., 1998) are minimized by choosing microwindows without significant $\mathrm{CO}_{2}$ and $\mathrm{HNO}_{3}$ Q-branches. FOV and ILS related effects have been shown to be of minor importance in radiative modelling for the comparable instrument MIPAS-ENVISAT by Stiller et al. (2002).

It has to be noted that the error resulting from the knowledge of the flight altitude can become important especially in ascent and descent phases, since the aircraft changes the flight altitude with several meters per second, and the GPS altitude information (which is also uncertain within several tens of meters) has to be interpolated to the mean time of an interferogram. Stray-light related errors were investigated by Keim (2002) for flights with strong particle contamination on the scan mirror of MIPAS-STR. During the 
RECONCILE campaign, no strong particle contamination of the scan mirror was observed and stray-light related effects were not identified. However, weak stray light contributions cannot be fully excluded. Significant errors resulting from the electronic data acquisition chain are not identified.

As described in Rodgers (2000), another relevant error may be the smoothing error. Since this error is highly dependent on the choice of the the estimate of the true ensemble covariance used for calculation (i.e. a climatological covariance matrix), we regard the retrieval result as an estimate of the smoothed version of the state and show also the vertical resolution.

For the retrieval of the LOS correction, in principle all errors listed above under "primary errors" are relevant for the determination of the individual LOS offsets. But since the LOS offset is estimated as the average of a large number of limb sequences (61 for RECONCILE flight 11), the different errors are expected to compensate each other. In Fig. 12, the retrieval results for the individual LOS offsets are shown for the whole flight. The values are scattered around a mean LOS offset of $5.45 \mathrm{arcmin}$ with a $1 \sigma$-uncertainty of 0.19 arcmin. Outliers can be caused by the availability of low numbers of quality-filtered observation geometries and/or different combinations of the individual error sources listed above. The outliers associated to the first sequence and the last sequences belong to ascent and descent phases, where the flight altitude is changing fast, resulting in additional uncertainties of the sampling geometries. The uncertainty of the corrected LOS is calculated taking into account the $1 \sigma$ uncertainties resulting from (i) the attitude information from the AHRS after postprocessing of $0.75 \mathrm{arcmin}$, (ii) errors resulting from the scan-mirror control of 0.09 arcmin and (iii) the $1 \sigma$-uncertainty of the derived mean LOS correction of $0.19 \mathrm{arcmin}$. The uncertainties are combined by the root of the square sum, resulting in an estimated LOS-uncertainty of 0.78 arcmin.

The error in the temperature retrieval is estimated according to Wetzel et al. (2002) for the balloon-borne MIPAS instrument. The effects of spectroscopic line data errors and uncertainties of the applied $\mathrm{CO}_{2}$-profile are estimated by retrievals with a shifted $\mathrm{CO}_{2}$-profile (5\%). LOS-related errors are estimated by retrievals taking into account of the LOSuncertainty ( $0.78 \mathrm{arcmin})$, and radiometric calibration errors are considered by retrievals with modified gain $(2 \%)$. The corresponding errors for retrieved temperature profiles are calculated as the differences between the retrieval results with the modified quantity and the initial retrieval results according to

$$
\begin{aligned}
& \Delta \boldsymbol{x}_{j}=\left(\begin{array}{l}
\Delta x_{1, j} \\
\Delta x_{2, j} \\
\vdots \\
\Delta x_{n_{\max }, j}
\end{array}\right) \\
& =\left(\mathbf{K}^{\mathrm{T}} \mathbf{S}_{y}^{-1} \mathbf{K}+\gamma \mathbf{L}^{\mathrm{T}} \mathbf{L}\right)^{-1} \mathbf{K}^{\mathrm{T}} \mathbf{S}_{y}^{-1}\left(\boldsymbol{y}_{\text {error }, j}-\boldsymbol{y}_{\text {result }}\right),
\end{aligned}
$$

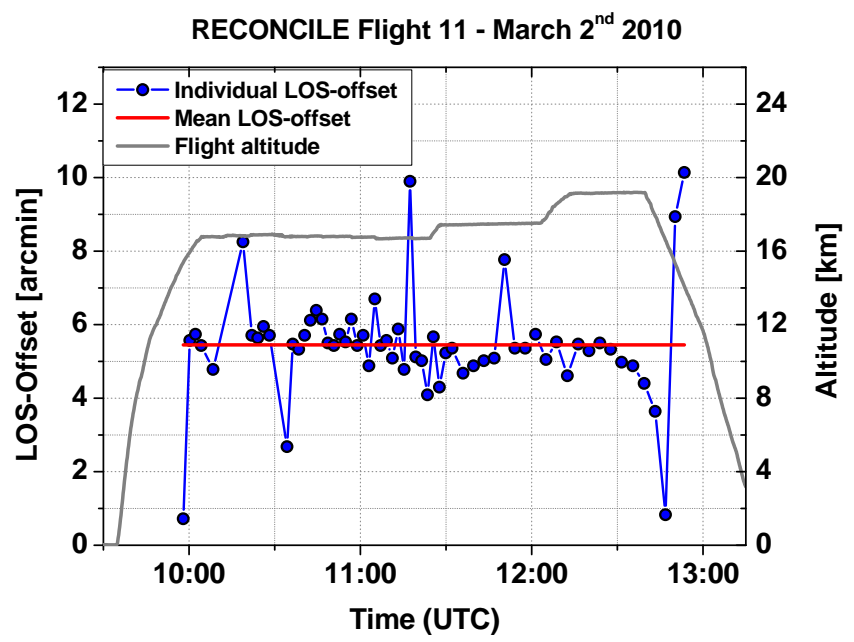

Fig. 12. Retrieved LOS offsets from the individual limb sequences and mean LOS offset used as LOS correction.

where $\Delta \boldsymbol{x}_{j}$ represents the vertical error profile resulting from the modified quantity $j, \Delta x_{n, j}$ the errors at the altitude levels $n, \boldsymbol{y}_{\text {error }, j}$ the calculated spectrum with the modified quantity and $\boldsymbol{y}_{\text {result }}$ the calculated spectrum of the initial retrieval result (von Clarmann, 2003). Together with the spectral noise error, the resulting error vectors are combined to the estimated $1 \sigma-$ uncertainty for temperature according to

$\Delta \boldsymbol{x}_{\text {temperature }}=\sqrt{\Delta x_{\text {prof }, \text { spec }}^{2}+\Delta x_{\text {los }}^{2}+\Delta x_{\text {cal }}^{2}+\Delta x_{\text {noise }}^{2}}$,

where $\Delta \boldsymbol{x}_{\text {temperature }}$ represents the estimated error vector of the retrieval result. The individual error contributions $\Delta \boldsymbol{x}_{\text {prof,spec }}, \Delta \boldsymbol{x}_{\text {los }}$ and $\Delta \boldsymbol{x}_{\text {cal }}$ result from the retrievals with the modified $\mathrm{CO}_{2}$-profile, LOS and gain, and $\Delta x_{\text {noise }}$ represents the spectral noise error. The estimated error of the retrieved temperature is used in the following retrievals for the determination of the corresponding temperature-related errors.

Following Wetzel et al. (2002), for the trace gas retrievals the spectroscopic errors (line data and cross-sections) are estimated to conservative constant percentages of the absolute profile values. The spectroscopic error of $8 \%$ for $\mathrm{HNO}_{3}$ is taken from Wetzel et al. (2002). Considering the results of Moore et al. (2006), for CFC-11 and CFC-12 spectroscopic errors of $10 \%$ are taken into account. For ozone, a spectroscopic error of $7 \%$, and for $\mathrm{H}_{2} \mathrm{O}$, a spectroscopic error of $10 \%$ is adopted, considering the uncertainties for the line intensities of the target signatures (Flaud et al., 2002, 2006 and references therein). For the $\mathrm{ClONO}_{2}$ cross-section, the worst case error of $5.5 \%$ reported by Wagner and Birk (2003) is considered. The errors for the trace gas retrievals due to the uncertainties of temperature, LOS and radiometric gain are also estimated according to Eq. (4) by retrievals with the corresponding modified quantities. The estimated $1 \sigma$-errors for the trace gas profiles are calculated according to 
$\Delta \boldsymbol{x}_{\mathrm{vmr}}=\sqrt{\Delta \boldsymbol{x}_{\mathrm{spec}}^{2}+\Delta \boldsymbol{x}_{\mathrm{temp}}^{2}+\Delta \boldsymbol{x}_{\mathrm{los}}^{2}+\Delta \boldsymbol{x}_{\mathrm{cal}}^{2}+\Delta \boldsymbol{x}_{\mathrm{noise}}^{2}}$,

with $\Delta \boldsymbol{x}_{\mathrm{vmr}}$ the estimated error vector of the retrieval result,

$\Delta \boldsymbol{x}_{\text {spec }}$ the spectroscopic error, $\Delta \boldsymbol{x}_{\text {temp }}$ the temperature related error, and the other contributions as in the case of the temperature error.

It has to be considered that the different error sources can show variations on different timescales. For example, while the noise error is characteristic for single spectra (recording of one interferogram takes approximately $9.5 \mathrm{~s}$ ), temperature-related errors can be relevant for full limb scans (a few minutes) and spectroscopic errors might affect entire flight sections. As a consequence, the combined error might show a more systematic behaviour in particular flight sections and the statistical combination of the errors has to be seen with suspicion. The discussed error-budget reflects the attempt to give one single error quantity summarizing all of the discussed effects.

The vertical resolution of the retrieval results is derived from the averaging kernel matrix A (Rodgers, 2000):

$\mathbf{A}=\left(\mathbf{K}^{\mathrm{T}} \mathbf{S}_{y}^{-1} \mathbf{K}+\gamma \mathbf{L}^{\mathrm{T}} \mathbf{L}\right)^{-1} \mathbf{K}^{\mathrm{T}} \mathbf{S}_{y}^{-1} \mathbf{K}$.

According to Purser and Huang (1993), the reciprocal of the peak of $\mathbf{A}$ can be taken as a measure of its width, which is often used as a measure of the vertical resolution of a retrieval result. We calculate the vertical resolution $\Delta a_{n}$ at the altitude $n$ as the absolute value of the quotient of the local vertical grid spacing $\Delta h_{n}$ divided by the trace element $\mathbf{A}_{n, n}$ :

$\Delta a_{n}=\left|\Delta h_{n} / \mathbf{A}_{n, n}\right|$.

The degrees of freedom (DOF) of the retrieval result are the sum of the trace elements of $\mathbf{A}$ and indicate how many independent pieces of information are derived from the measurements allowed by the regularization (e.g. Steck, 2002).

In the left panel of Fig. 13, the retrieval result for a single $\mathrm{HNO}_{3}$ profile is shown together with the estimated $1 \sigma$ error. The initial guess/a priori profile used for the retrieval is also shown. The retrieval result is very distinct from the initial guess/a priori profile and reflects the weak regularization strength. In the retrieved profile, a local $\mathrm{HNO}_{3}$-maximum at about $15 \mathrm{~km}$ is identified, which can be assigned to reand de-nitrification layers at the edge of the polar-vortex (see Sect. 7.3).

The individual contributions of the different error sources are shown in the middle panel. Between flight altitude and the lowest tangent point, the dominating error source is the uncertainty of the spectroscopic data, followed by the errors resulting from the radiometric calibration and the uncertainties of the retrieved temperature profiles. The LOS error is variable with altitude, and is together with the spectral noise error of minor importance. For sequences with more extended vertical coverage, all estimated errors except that

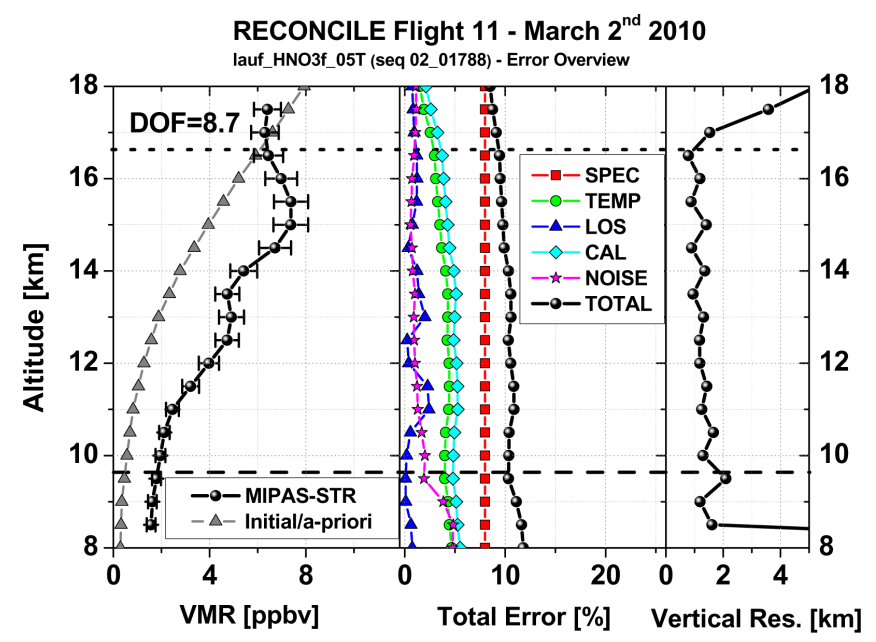

Fig. 13. Left side: retrieved vertical profile of $\mathrm{HNO}_{3}$ with estimated error and initial guess/a priori profile (DOF=degrees of freedom). Middle: Different error contributions and estimated error in percent $(\mathrm{SPEC}=$ spectroscopic data, TEMP $=$ temperature, $\mathrm{LOS}=$ line-ofsight, $\mathrm{CAL}=$ radiometric calibration, NOISE $=$ spectral noise, TOTAL $=$ estimated $1 \sigma$-error). Right side: Vertical resolution of the retrieval result. The dotted horizontal line indicates the flight altitude of the Geophyisca and the dashed horizontal line the lowest tangent point of the scan.

of the assumed spectroscopic line data error significantly increase towards the lower observation geometries, and the LOS error and the spectral noise error can become the dominating errors. The relative importance of the different errors can vary from scan to scan, depending on the atmospheric situation, the vertical distribution of the target species, and the sampling geometries.

For the result shown, the estimated error is between 9 to $12 \%$ in the altitude range between flight altitude and the lowest tangent point. Above flight altitude, the absolute numbers of all errors except of the spectroscopic error still decrease virtually, but this has to be interpreted in the context of the vertical resolution indicated in the right panel. For the measurement geometries between flight altitude and the lowest tangent point, a high vertical resolution of mainly between 1 and $1.5 \mathrm{~km}$ is achieved due to the availability of considerable vertical information included in the spectra with negative elevation angles. Therefore, the estimated error is representative in this altitude range in the context of the vertical resolution. From the geometries with positive elevation angles, mainly column information is obtained for the atmosphere above the aircraft, and the vertical resolution rapidly decreases. Hence, for the parts of the retrieved profiles above the aircraft, the absolute numbers of the errors still decrease, but the vertical information is "smeared" over a broader vertical range. Practically, only column information is obtained above $17.5 \mathrm{~km}$ and the errors become physically meaningless. At altitudes below approximately $8.5 \mathrm{~km}$, which are not covered by the 


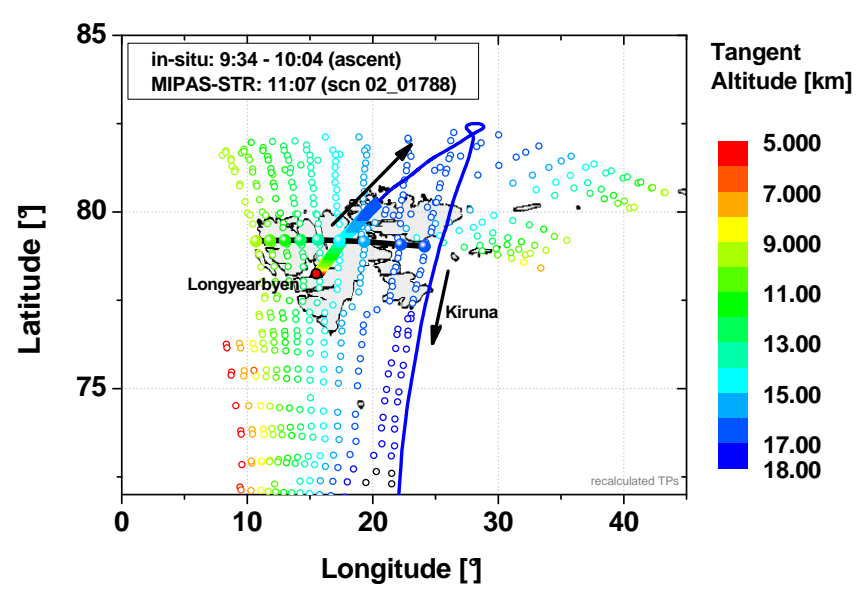

Fig. 14. Limb scan of MIPAS-STR selected for comparisons with in-situ profiles. The distribution of the tangent points of MIPASSTR (circles) along the flight path (blue line) is indicated colourcoded with reference to the tangent altitude. The selected scan 02_01788 for the comparisons is indicated by filled circles. The flight path of the Geophysica is plotted colour-coded with altitude for the ascent phase.

measurements, no vertical information is obtained, as indicated by the vertical resolution tending towards infinity. For the $\mathrm{HNO}_{3}$-profile shown in Fig. 13, 8.7 DOF are obtained, indicating weak influence of the regularization (for sampling details of the shown scan 02_01788 see Sect. 7.1).

\section{Results}

\subsection{Approach for comparison of MIPAS-STR results and in-situ measurements}

Several in-situ instruments were deployed onboard the Geophysica during the RECONCILE campaign, allowing for comparisons with the MIPAS-STR results (Table 3). High precision ambient temperature measurements were obtained from the Thermodynamic Complex (TDC). Ozone measurements were taken by the Fast-Response Chemiluminescent Airborne Ozone Analyzer (FOZAN). CFC-11 and CFC-12 measurements were provided by the High Altitude Gas AnalyzeR (HAGAR). Gas-phase water vapour measurements were obtained by the Fluorescent Lyman-Alpha Stratospheric Hygrometer for Aircraft (FLASH-A), which is a recent modification of the previously utilized FLASH instrument on board of the Geophysica aircraft (Sitnikov et al., 2007).

For the discussed flight, MIPAS-STR measurements of high spatial coincidence with the in-situ measurements related to the ascent phase of the Geophyisca are available (Fig. 14). The lowest tangent point of the selected scan 02_01788 is located at an altitude of $9.7 \mathrm{~km}$ and the flight altitude is $16.7 \mathrm{~km}$, whereas 9 limb-viewing and 2 upward- viewing geometries were carried out. The tangent point approximately coinciding with the flight path during ascent corresponds to a tangent altitude of $14.5 \mathrm{~km}$. For comparisons, it has to be considered that the air volume sampled by MIPAS-STR during one limb sequence (duration typically a few minutes) covers horizontally several tens of kilometres along flight track and several hundreds of kilometres along the viewing direction (see Sect. 3), and the vertical resolution is limited compared to in-situ measurements. In contrast, the in-situ measurements giving rise to the ascent profile are carried out in a more extended time interval (about half an hour) and provide precise measurements at certain altitudes and positions. The limited temporal coincidence (time mismatch ca. $1 \mathrm{~h}$ ) between the MIPAS-STR measurements and the in-situ measurements also has to be kept in mind, since chemical and dynamical atmospheric structures can move or change in the time between the measurements.

The retrieval results at flight altitude are compared to the in-situ measurements along the flight track for flight sections with approximately constant flight altitude. Three different flight sections with approximately constant flight altitude are situated between 10:05-11:25 (I) UTC, 11:2512:05 (II) UTC and 12:05-12:45 (III) UTC (Fig. 6). The insitu measurements are compared to the retrieval results at the grid-altitudes 17.0, 17.5 and $19 \mathrm{~km}$, respectively.

As discussed in Sect. 5, the edge of the late Canadian polar vortex remnant was located above Spitsbergen at the date of RECONCILE flight 11. During the ascent phase, the insitu instruments subsequently sampled air below and inside the vortex. During the recording of scan 02_01788, MIPASSTR was pointing into a region at the vortex edge, partially overlapping with the in-situ ascent profile (compare Figs. 8 and 14). At higher profile altitudes the in-situ measurements are located closer to the core region of the vortex remnant compared to the MIPAS-STR tangent points.

\subsection{Retrieval results and in-situ comparison}

The retrieval results for scan 02_01788 of MIPAS-STR are shown together with the corresponding in-situ measurements in Fig. 15a-f. In the case of $\mathrm{ClONO}_{2}$, no suitable in-situ measurements are available for comparisons, but the retrieval result is also discussed for completeness. For the retrieved profiles, the estimated $1 \sigma$-error, vertical resolution and DOF are indicated. Retrieved profiles and in-situ results are shown with reference to geometric (GPS-) altitude. Adapted ECMWF-data (temperature and pressure) refer to geopotential height, which is comparable to geometric altitude in the considered vertical range to a good approximation.

For the in-situ measurements, profiles smoothed with the corresponding averaging kernels of MIPAS-STR are also shown (Rodgers, 2000). This approach allows for the comparison of the results of MIPAS-STR with the in-situ measurements at the common vertical resolution of MIPAS-STR. In the case of temperature, also the interpolated profile from 
Table 3. In-situ instruments onboard the Geophysica used for comparisions with MIPAS-STR.

\begin{tabular}{lclcl}
\hline Instrument & Target & $\begin{array}{l}\text { Time } \\
\text { resolution }\end{array}$ & Accuracy & Reference \\
\hline TDC & Temperature & $1 \mathrm{~s}$ & $0.5 \mathrm{~K}$ & Shur et al. (2006) \\
FOZAN & $\mathrm{O}_{3}$ & $2 \mathrm{~s}$ & $<10 \%$ & Ulanovsky et al. (2001) \\
HAGAR & $\mathrm{CFC}-11$ & $90 \mathrm{~s}$ & $0.5-5 \mathrm{ppt}(1.4-5.6 \%)$ & $\begin{array}{l}\text { Riediger et al. (2000) } \\
\text { Werner et al. (2010) }\end{array}$ \\
HAGAR & CFC-12 & $90 \mathrm{~s}$ & $2-8 \mathrm{ppt}(1.2-1.7 \%)$ & $\begin{array}{l}\text { Riediger et al. (2000) } \\
\text { Werner et al. (2010) }\end{array}$ \\
& & & $10 \%$ & see text \\
\hline
\end{tabular}

ECMWF is shown, which serves as initial guess/a priori profile for the retrieval. For the trace gases, the corresponding climatological profiles serving as the initial guess/a priori profiles are also shown. For all profiles of MIPAS-STR shown in Fig. 15a-f, only profile points with a vertical resolution of better than $5 \mathrm{~km}$ are plotted.

As can be seen in Fig. 15a for temperature, the agreement of MIPAS-STR and TDC is mostly better than $1 \mathrm{~K}$ and the agreement is improved compared to the interpolated ECMWF-profile. In the upper part of the profile, the agreement between MIPAS-STR and TDC slightly decreases, which is attributed to the decreasing spatial overlap of the measurements. For $\mathrm{O}_{3}$, also reasonable agreement is found between MIPAS-STR and FOZAN, as can be seen in Fig. 15b. The unsmoothed profile from FOZAN shows variable mixing ratios indicating horizontal structures in the $\mathrm{O}_{3}$ distribution, especially between 12 and $14 \mathrm{~km}$. In this altitude range, the retrieved profile from MIPAS-STR follows the upper part of the in-situ profile, while this structure is not apparent in the smoothed in-situ profile.

As can be seen in Fig. 15c and d for CFC-11 and CFC-12, the results from MIPAS-STR and HAGAR are also mostly in agreement within $1 \sigma$ of the estimated error below $15 \mathrm{~km}$ and $16 \mathrm{~km}$, respectively. At higher altitudes, which are characterized by very low mixing ratios of the CFCs, the results start to diverge. This finding is attributed to the fact that the horizontally extended MIPAS-STR measurements here are significantly affected by spectral contributions from extravortex air with much higher mixing ratios of the CFCs. As will be shown below, the CFCs show by far the strongest contrasts between vortex and extra-vortex air from the trace gases considered for in-situ comparisons here.

In Fig. 15e, the results for $\mathrm{H}_{2} \mathrm{O}$ from MIPAS-STR and FLASH-A are shown, and the instruments agree within their individual uncertainties. Slightly increasing differences below $10 \mathrm{~km}$ are attributed to the decreasing spatial overlap of the measurements and the tropospheric variability of $\mathrm{H}_{2} \mathrm{O}$. The retrieved profile of $\mathrm{ClONO}_{2}$ is shown in Fig. 15f, where no in-situ measurements suitable for comparisons are available. The retrieved profile indicates high $\mathrm{ClONO}_{2}$-mixing ratios in the upper part of the profile, characteristic for the chlorine-deactivated air in the late vortex. Virtually negative mixing ratios below $10 \mathrm{~km}$ are a consequence of very low mixing ratios of $\mathrm{ClONO}_{2}$ in the troposphere and can result from spectral interference with other species and smoothing effects of the retrieval. The corresponding retrieved profile for $\mathrm{HNO}_{3}$ (for which also no in-situ comparison is performed) is discussed in Sect. 6.5 (Fig. 13).

For the shown retrieved profiles of the discussed scan, the estimated $1 \sigma$-errors are typically about $10-15 \%$. Typical vertical resolutions of 1 to $2 \mathrm{~km}$ are achieved in the altitude range of the tangent points. Between 6.4 and 8.7 DOF are obtained from the 9 limb-viewing and 2 upward-viewing geometries, indicating considerable vertical information included in the measurements. For the continuum retrievals (not shown here) carried out for each microwindow simultaneously together with the target parameters, typically about 7 DOF are obtained for this scan. The shown retrieval results show very low sensitivity on the initial guess/a priori information as a consequence of the high signal-to-noise ratios of the measurements and the applied weak regularization.

The comparison of the retrieval results and the in-situ measurements along the flight track is shown in Fig. 16ae. The retrieval results of MIPAS-STR for temperature are in very good agreement with the results of TDC (Fig. 16a) and the agreement is improved compared to the interpolated ECMWF temperatures. As can be seen in Fig. 16b, for $\mathrm{O}_{3}$ also good agreement is found between MIPAS-STR and FOZAN.

The results of MIPAS-STR for CFC-11 and CFC-12 along the flight track are also in principle in agreement with the measurements of HAGAR (Fig. 16c and d), often already within $1 \sigma$ of the errors. As in the case of the profile comparisons, higher mixing ratios of the CFCs are found in the first flight section for the MIPAS-STR results at flight altitude and are attributed to spectral contributions from outside of the polar vortex. The strong contrasts of the mixing ratios of the CFCs at the vortex edge can be seen consistently in the MIPAS-STR and HAGAR results in Fig. 16c and $d$. The vortex edge shows up in the HAGAR measurements sharply between 11:14 UTC and 11:21 UTC by strong increases of the mixing ratios of CFC-11 and CFC-12 of $80 \mathrm{pptv}$ and $180 \mathrm{pptv}$, respectively. In the case of MIPASSTR, the corresponding structures appear more smoothly 

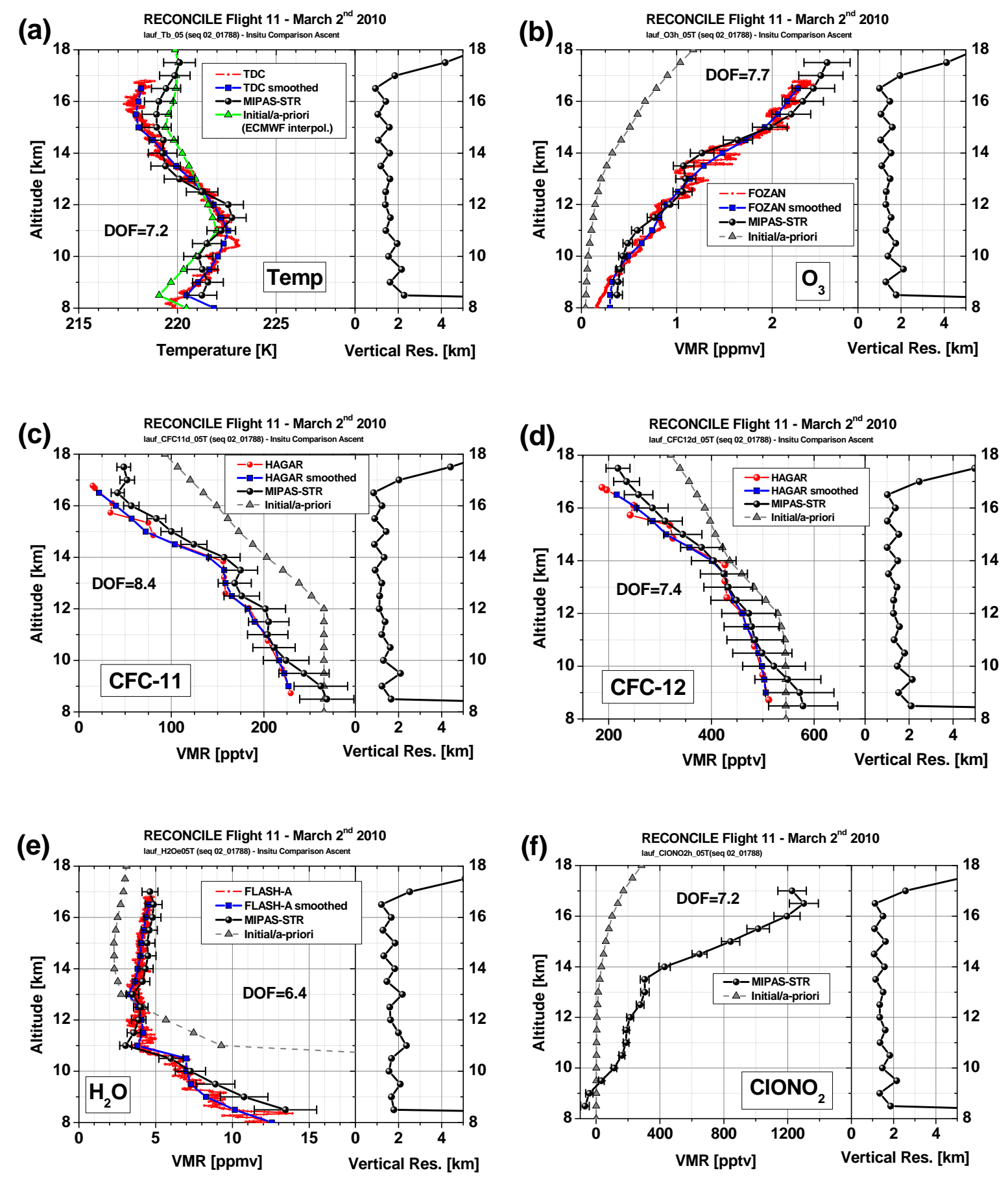

Fig. 15. (a-f): Retrieved profiles of temperature, $\mathrm{O}_{3}, \mathrm{CFC}-11, \mathrm{CFC}-12, \mathrm{H}_{2} \mathrm{O}$ and $\mathrm{ClONO}_{2}$ with estimated error and comparison with in-situ measurements (left-hand side panels in the plots). Initial guess/a priori profiles for the retrievals and DOF of the results are also indicated. Vertical resolutions of the retrieval results (right-hand side panels in the plots).

between 11:00 UTC and 11:25 UTC as a consequence of the different sampling characteristics. For comparison, the differences between vortex and extra-vortex air are less pronounced in the case of $\mathrm{O}_{3}$ along the flight track, as consistently indicated by the results of MIPAS-STR and FOZAN (Fig. 16b). For CFC-11 and CFC-12, noticeable differences between the results of MIPAS-STR and HAGAR are found also especially in the last flight section (12:15-12:45 UTC) (Fig. 16c and d). The lower mixing ratios in the results of MIPAS-STR are explained by the fact that filaments of vortex air with lower mixing ratios of the CFCs were located along the instruments viewing direction in this particular flight part 

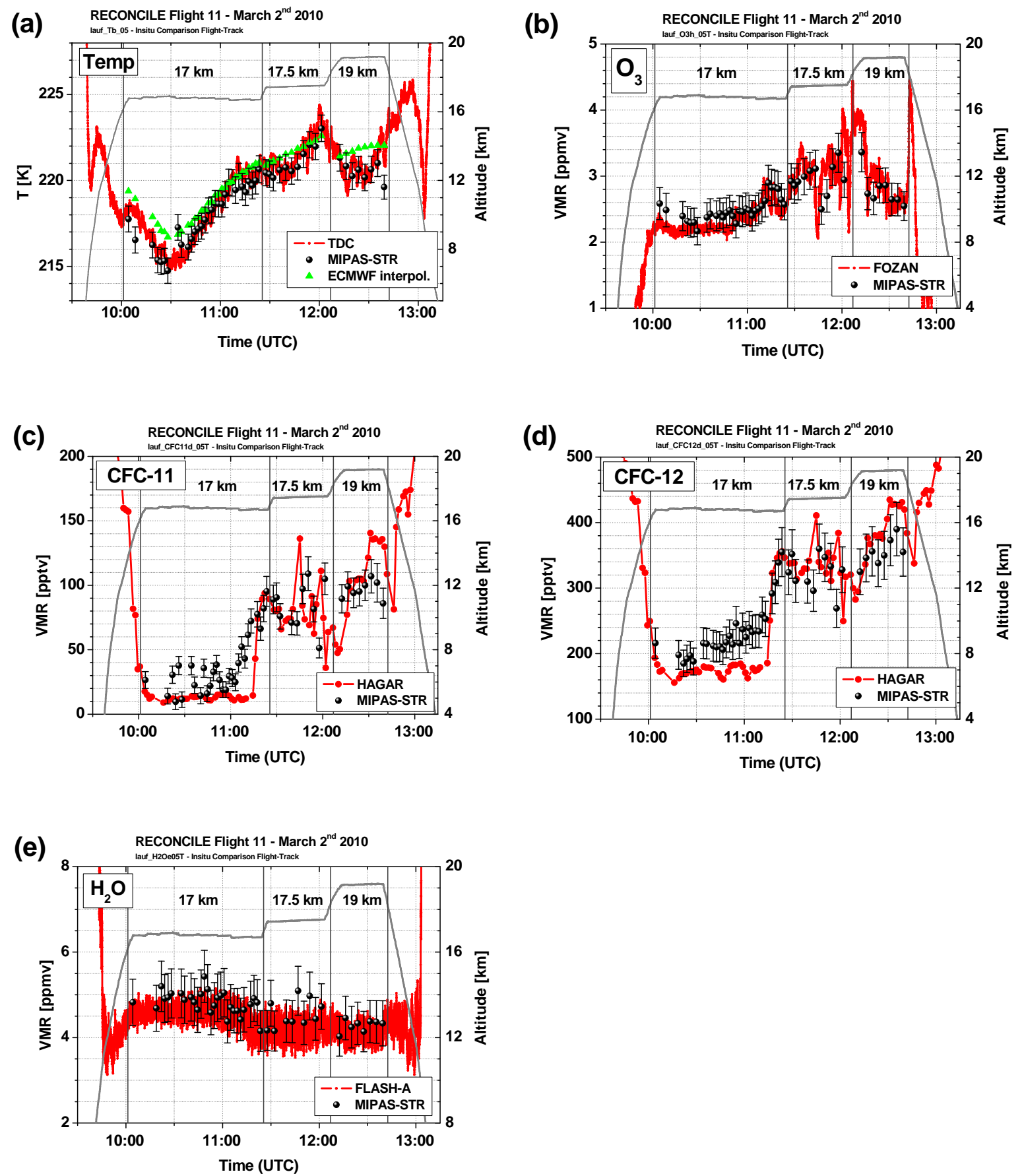

Fig. 16. (a-e): Comparison of the retrieval results inclusive estimated error for temperature, $\mathrm{O}_{3}, \mathrm{CFC}-11, \mathrm{CFC}-12$ and $\mathrm{H}_{2} \mathrm{O}$ with in-situ measurements along flight track. Retrieval results are shown for the indicated retrieval-grid altitudes.

(compare Fig. 8 and Sect. 7.3), contributing to the spectra of MIPAS-STR. As can be seen in Fig. 16e for $\mathrm{H}_{2} \mathrm{O}$, agreement is found between the results of MIPAS-STR and FLASH-A.

The mean differences and the sample standard deviations of the mean differences between the results of MIPAS-STR and the in-situ measurements for the shown profile comparisons and comparisons along the flight track are summarized in Table 4. While the mean differences indicate systematic differences between the MIPAS-STR and the in-situ results, the sample standard deviations of the mean differences indicate the qualitative agreement for single retrieval-grid points. Both quantities together indicate the quantitative agreement. In the case of the profile comparisons, these quantities are calculated taking into account the smoothed in-situ results at 


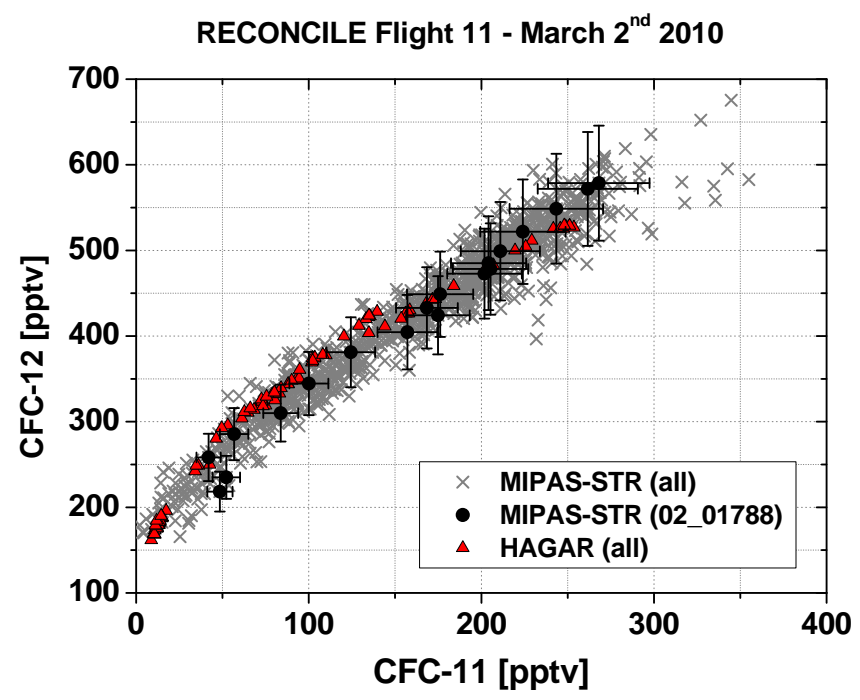

Fig. 17. Correlation of CFC-11 and CFC-12 derived from the MIPAS-STR and HAGAR measurements for RECONCILE flight 11. For MIPAS-STR, all retrieved profile points with a vertical resolution better than $5 \mathrm{~km}$ are shown. The points associated to sequence 02_01788 of MIPAS-STR, for which the in-situ profile-comparisons are carried out, are shown together with the corresponding estimated $1 \sigma$-errors.

the retrieval-grid altitudes. For the comparisons along flight track, the unsmoothed in-situ results are interpolated in time for the MIPAS-STR retrieval results.

With mean differences of $+0.2 /-0.2 \mathrm{~K}$ for the profile and along-flight-track comparison, and respective sample standard deviations of about $0.7 / 0.6 \mathrm{~K}$, the retrieved temperatures of MIPAS-STR agree very well with the TDC measurements within the uncertainties of the involved instruments. Also for $\mathrm{O}_{3}$ excellent agreement is found between MIPASSTR and FOZAN. The absolute mean differences between MIPAS-STR and HAGAR for CFC-11 and CFC-12 in the range of $8-21$ pptv are also small. However, all mean differences calculated for the CFCs are positive, which is attributed to the extended first flight section with the MIPASSTR measurements significantly biased by extra-vortex air. The corresponding percentages of the sample standard deviations of the mean differences (see Table 4) reflect the fact that the strong horizontal contrasts in the mixing ratios of the CFCs alter the comparability of the MIPAS-STR and HAGAR measurements. These comparably high values are mainly driven by the differences at high profile altitudes with very low absolute mixing ratios of the CFCs. However, this is masking the fact that the absolute mean differences and sample standard deviations for the CFCs are in the low ppt-range, indicating considerable agreement at higher mixing ratios of the CFCs (i.e. at lower profile altitudes). For the profile/along-flight-track comparisons for $\mathrm{H}_{2} \mathrm{O}$, the mean differences and corresponding sample standard deviations indicate agreement between the involved instruments.

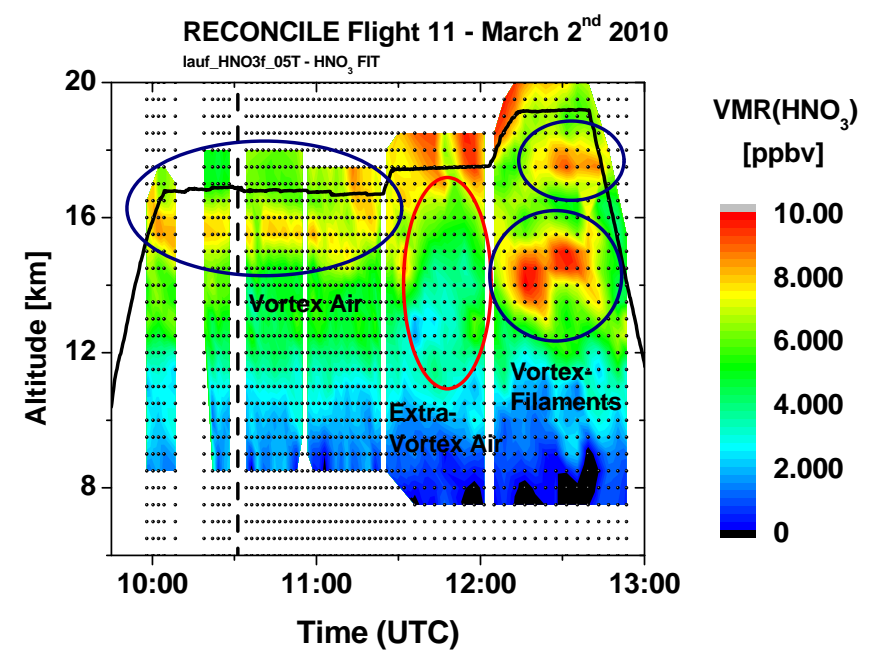

Fig. 18. Vertical cross-section of $\mathrm{HNO}_{3}$ along flight track derived from MIPAS-STR measurements. The turn between the northeastern flight leg (from Longyearbyen) to the southward flight leg (towards Kiruna) is indicated by the dashed vertical line. Retrieved $\mathrm{HNO}_{3}$-mixing ratios are linearly interpolated between the retrieval grid points (black dots) and are shown colour-coded. Interpolation is performed between grid points with vertical resolutions of the result of better than $5 \mathrm{~km}$.

To overcome the complications of the limited spatial overlap of the MIPAS-STR and HAGAR results in the context of the strong horizontal contrasts of the mixing ratios of the CFCs, we show the correlations of CFC-11 and CFC-12 derived from MIPAS-STR and HAGAR in Fig. 17. Collocated pairs of CFC-11 and CFC-12 measurements of the individual instruments are considered, whereas no spatial coincidence between the measurements of the two instruments is necessary. The resulting correlations show a high degree of agreement within the variability of the results of MIPAS-STR and the associated uncertainties. Only for mixing ratios of CFC11 between 30 and 150 pptv, slightly higher mixing ratios of CFC-12 are found for HAGAR, but the corresponding results are still within the variability of the MIPAS-STR results.

\subsection{Two dimensional trace gas distributions}

The retrieved vertical trace gas profiles obtained from the MIPAS-STR measurements are combined to 2-dimensional vertical cross-sections of the corresponding species along the flight track. The resulting vertical cross-section for $\mathrm{HNO}_{3}$ is shown in Fig. 18. In the first flight section between 10:00 UTC and 11:15 UTC, at altitudes above $14.5 \mathrm{~km}$, air of the late vortex can be clearly identified. A weak renitrification remnant is found with a $\mathrm{HNO}_{3}$-maximum at $15.5 \mathrm{~km}$ and mixing ratios of about $8 \mathrm{ppbv}$, indicating between 2 to 3 ppbv higher mixing ratios compared to the layers above and below. The resolved vertical thickness of the layer is about $1 \mathrm{~km}$, which is close to the vertical resolution limit 


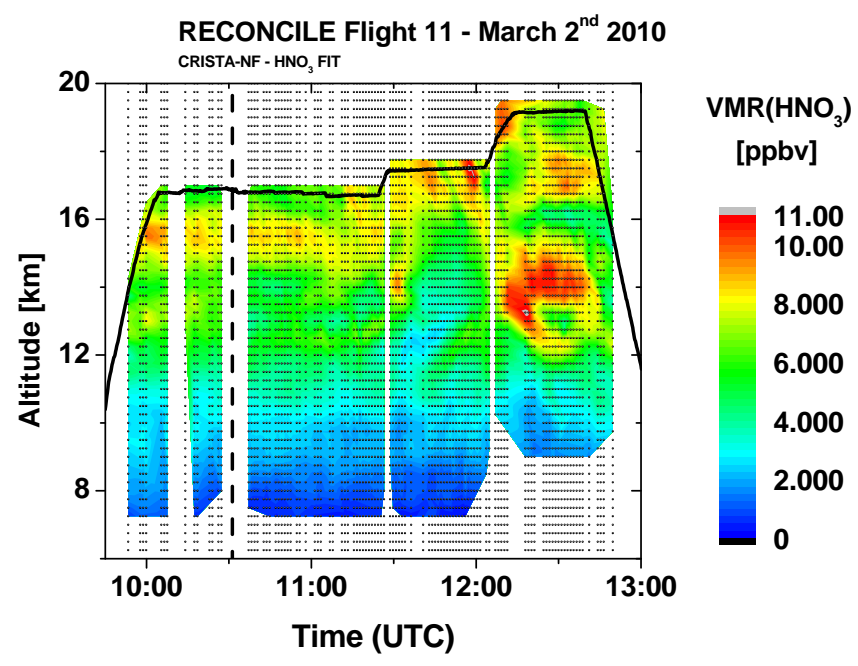

Fig. 19. Vertical cross-section of $\mathrm{HNO}_{3}$ along flight track derived from CRISTA-NF measurements (compare Fig. 18).

of MIPAS-STR. Above, denitrified air characterised by low $\mathrm{HNO}_{3}$-mixing ratios is found.

Between 11:35 UTC and 12:00 UTC, a structure of extra vortex air can be identified, which is characterized by low $\mathrm{HNO}_{3}$-mixing ratios below $16 \mathrm{~km}$ and shows no de/renitrification structure. The structure is diagonally linked to further structures of extra-vortex air in the last flight section with low $\mathrm{HNO}_{3}$-mixing ratios, characteristic for extravortex air. In the last flight section, between 12:05 UTC and 12:45 UTC, two prominent structures with strongly enhanced $\mathrm{HNO}_{3}$-mixing ratios are apparent, which can be assigned to filaments of the polar vortex with renitrified air. $\mathrm{HNO}_{3}$ mixing ratios of up to $10 \mathrm{ppbv}$ are found for the lower structure centred at $14.5 \mathrm{~km}$ with a vertical extension of about $3 \mathrm{~km}$, whereas the mixing ratio is increased by about 5 ppbv compared to the surrounding air. The tiny upper structure located around $17.5 \mathrm{~km}$ with a vertical extension of about $1.5 \mathrm{~km}$ also shows significantly enhanced $\mathrm{HNO}_{3}$ mixing ratios of up to 9 ppbv.

In Fig. 19, the corresponding vertical cross-section of $\mathrm{HNO}_{3}$ derived from CRISTA-NF measurements is shown. CRISTA-NF is capable of measuring limb-emission spectra in a comparable spectral region like MIPAS-STR and is pointing approximately into the same direction. The characteristics of CRISTA-NF are briefly summarized in Table 5 (e.g. Spang et al., 2008; Ungermann et al., 2011). While MIPAS-STR with its comparable high spectral resolution is able to separate weak trace gas signatures not resolved by CRISTA-NF, the advantage of CRISTA-NF is the higher vertical and horizontal sampling density allowing for the identification of atmospheric fine-structures not resolved by MIPAS-STR.

$\mathrm{HNO}_{3}$ is retrieved from the CRISTA-NF measurements in the same spectral region as for MIPAS-STR and a typical

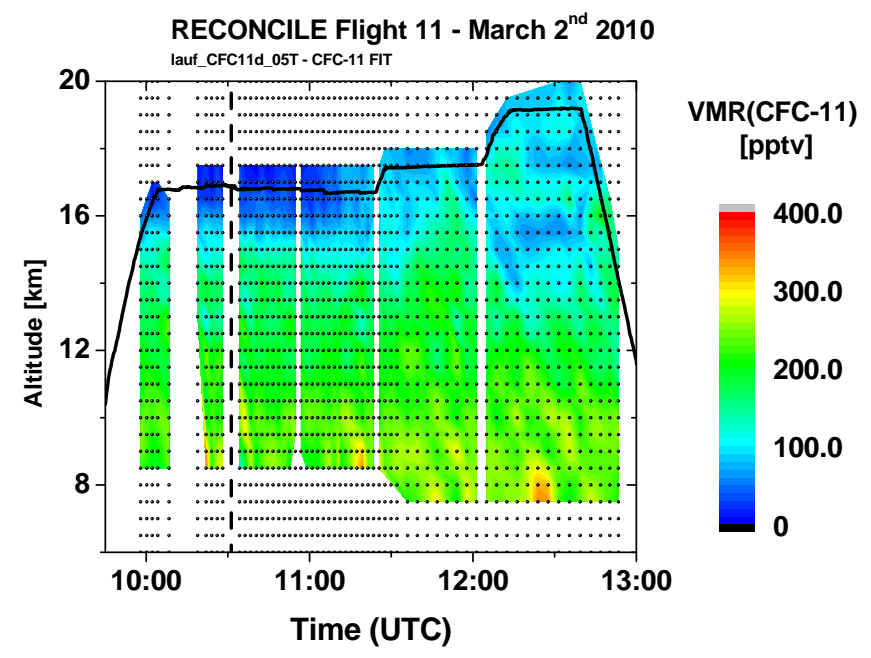

Fig. 20. Vertical cross-section of CFC-11 along flight track derived from MIPAS-STR measurements (compare Fig. 18).

vertical resolution of $0.5 \mathrm{~km}$ is obtained. For details concerning the CRISTA-NF retrieval see Ungermann et al. (2011). As can be seen in Figs. 18 and 19, the retrieved vertical cross-sections of $\mathrm{HNO}_{3}$ from the two instruments agree very well. All major structures observed in the MIPAS-STR result are also identified for CRISTA-NF: the de- and renitrification layers in the first part of the flight, the structure with extra-vortex air in the middle of the flight, and the vortex filaments in the last part of the flight. Several smaller substructures can also be identified in both results. It has to be noted that the range of the colour-coding for CRISTA-NF in Fig. 19 is slightly higher than for MIPAS-STR in Fig. 18. Higher peak values observed in the CRISTA-NF result are consistent with atmospheric fine-structures, with enhanced $\mathrm{HNO}_{3}$-mixing ratios spatially higher resolved by CRISTANF. However, we emphasize that the measurements of the two limb-sounding instruments using different techniques and processed with different forward and inversion models do result in cross-sections with considerable agreement.

The vertical cross-section of CFC-11 from MIPAS-STR in Fig. 20 shows a complementary picture to the $\mathrm{HNO}_{3}$ crosssections: Vortex air in the first flight section and vortex filaments at the end of the flight are characterised by low mixing ratios of CFC-11, while extra vortex air is indicated by increased mixing ratios of CFC-11 at stratospheric altitudes.

Some of the structures visible in the cross-sections of $\mathrm{HNO}_{3}$ and CFC-11 are horizontally largely extended (like the renitrification remnant in the beginning, with an extension along flight track of about $500 \mathrm{~km}$ between the turning point and the vortex edge) or show diagonally linked patterns and further fine-structures. 
Table 4. Mean differences and sample standard deviations ( $1 \sigma$, in parentheses) of the mean differences between MIPAS-STR and in-situ results for profile comparison and comparison along flight track.

\begin{tabular}{|c|c|c|c|c|}
\hline Target & \multicolumn{2}{|c|}{ Vertical profile } & \multicolumn{2}{|c|}{ Flight track } \\
\hline Temperature & $0.21(0.73) \mathrm{K}$ & & $-0.19(0.64) \mathrm{K}$ & \\
\hline $\mathrm{O}_{3}$ & $-0.01(0.12) \mathrm{ppmv}$ & $-3(14) \%$ & $0.05(0.26) \mathrm{ppmv}$ & $2(9) \%$ \\
\hline CFC-11 & $17(9)$ pptv & $14(13) \%$ & $8(24) \mathrm{pptv}$ & $21(40) \%$ \\
\hline CFC-12 & 21(19) pptv & $5(5) \%$ & 14 (42) pptv & $8(16) \%$ \\
\hline $\mathrm{H}_{2} \mathrm{O}$ & $1.16(1.91) \mathrm{ppmv}$ & $8(14) \%$ & $0.29(0.36) \mathrm{ppmv}$ & $6(7) \%$ \\
\hline
\end{tabular}

Table 5. Characteristics of CRISTA-NF. The spectral resolution is given for the low resolution spectrometer (LRS) which is capable of detecting $\mathrm{HNO}_{3}$.

\begin{tabular}{ll}
\hline \multicolumn{2}{c}{ CRISTA-NF } \\
\hline Spectrometer type & 2 Ebert-Fastie \\
& Grating Spectrometers \\
Spectral range & $667-2500 \mathrm{~cm}^{-1}$ \\
Spectral resolution & $1.6 \mathrm{~cm}^{-1}(\mathrm{LRS})$ \\
FOV (vertical x horizontal) & $3 \times 300 \mathrm{arcmin}$ \\
Vertical/horizontal sampling density & $0.25 / 15 \mathrm{~km}$ \\
Time per spectrum/limb scan & $\sim 1.2 / 70 \mathrm{sec}$ \\
\hline
\end{tabular}

\section{Conclusions}

In the work in hand, we present the FTIR limb-sounder MIPAS-STR deployed on the high altitude aircraft M55 Geophysica in its current performance. The applied calibration scheme from the measured data towards radiometrically calibrated spectra is discussed. A comprehensive bottom-up retrieval scheme for the reconstruction of temperature, $\mathrm{HNO}_{3}$, $\mathrm{O}_{3}$, CFC-11, CFC-12, $\mathrm{ClONO}_{2}$ and $\mathrm{H}_{2} \mathrm{O}$ is introduced, including the correction of a systematic line-of-sight offset. The significant influences of continuum-like effects on limbemission mid-infrared spectra in the UTLS region and their treatment by the retrieval of wavenumber-independent background continuum on the scale of retrieval microwindows are discussed. Retrieval results are shown exemplary for RECONCILE flight 11 on 2 March 2010.

The estimated overall $1 \sigma$-errors of the retrieval results are typically below $1 \mathrm{~K}$ for temperature and between 10 and $15 \%$ for the trace gases (including spectroscopic errors) in the vertical range spanned by the tangent points and directly above the flight altitude. In this region, characteristic vertical resolutions of 1 to $2 \mathrm{~km}$ are obtained, allowing for the identification of narrow vertical structures.

The retrieval results show a high degree of consistency with collocated in-situ measurements, taking into account the errors of MIPAS-STR and the in-situ results, the vertical and horizontal resolution of the MIPAS-STR results, atmospheric inhomogeneities and the geographical and time-mismatch between the MIPAS-STR and in-situ measurements. Higher discrepancies apparent for CFC-11 and CFC-12 around flight altitude are attributed to the observed strong contrasts in the mixing ratios of the CFCs between vortex and extra-vortex air. The comparison of the correlations of CFC-11 and CFC12 derived from the MIPAS-STR and HAGAR measurements, respectively, undelines the good agreement between the two instruments.

In the retrieved vertical cross-sections of $\mathrm{HNO}_{3}$ and $\mathrm{CFC}$ 11 , detailed mesoscale structures are identified, which are attributed to vortex air, vortex filaments and extra-vortex air. Small-scale structures with vertical extensions down to $1 \mathrm{~km}$ are resolved and are well in line with results from the infrared limb-sounder CRISTA-NF.

The presented results give insights into the properties of the Arctic UTLS in early 2010 and demonstrate the suitability of MIPAS-STR for quantitative studies of the chemistry and dynamics of the UTLS region with rather high spatial resolution. This allows for further studies with MIPAS-STR data on mesoscale structures, denitrification processes and chlorine activation.

Acknowledgements. We dedicate this paper to C. E. Blom, who greatly contributed to the success of MIPAS-STR onboard the M55 Geophyisca over many years. This work was supported by the EU under the grant number RECONCILE-226365-FP7-ENV-2008-1. We thank the RECONCILE coordination team, Myasishchev Design Bureau and Enviscope for making the RECONCILE field campaign a success. ECMWF data for the MIPAS-STR retrievals were obtained via NILU's NADIR database. We acknowledge support by Deutsche Forschungsgemeinschaft and Open Access Publishing Fund of Karlsruhe Institute of Technology. W. Woiwode thanks A. Kleinert and G. Wetzel from IMK-ASF, Karlsruhe Institute for Technology, for helpful discussions, and M. von Hobe from IEK-7, Research Centre Jülich, as representative of the RECONCILE team for especially supporting young scientists.

Edited by: H. Worden 


\section{References}

Andreae, M. O. and Crutzen, P. J.: Atmospheric Aerosols: Biogeochemical Sources and Role in Atmospheric Chemistry, Science, 276, 1052-1058, 1997.

Arnold, F. and Bührke, Th.: New $\mathrm{H}_{2} \mathrm{SO}_{4}$ and $\mathrm{HSO}_{3}$ vapour measurements in the stratosphere - evidence for a volcanic influence, Nature, 301, 293-295, 1983.

Blom, C. E., Gulde, T., Keim, C., Kimmig, W., Piesch, C., Sartorius, C., and Fischer, H.: MIPAS-STR: Entwicklung eines Instruments für Stratosphärenflugzeuge, Statusseminar des Ozonforschungsprogramms, 1998.

Blom, C. E., Camy-Peyret, C., Catoire, V., Chance, K., Oelhaf, H., Ovarlez, J., Payan, S., Pirre, M., Piesch, C., and Wetzel, G: Validation of MIPAS temperature profiles by stratospheric balloon and aircraft measurements, Proc. ACVE-2 meeting, 3-7 May 2004, Frascati, Italy, 2004.

Boone, C. D., Walker, K. A., and Bernath, P. F.: Speeddependent Voigt profile for water vapor in infrared remotesensing applications, J. Quant. Spectrosc. Ra., 105, 525-532, doi:10.1016/j.jqsrt.2006.11.015, 2007.

Brault, J. W.: New approach to high precision Fourier transform spectrometer design, Appl. Opt., 35, 2891-2896, 1996.

Ciurylo, R.: Shapes of pressure- and Doppler-broadened spectral lines in the core and near wings, Phys. Rev. A, 58, 1029-1039, 1998.

Cortesi, U., Lambert, J. C., De Clercq, C., Bianchini, G., Blumenstock, T., Bracher, A., Castelli, E., Catoire, V., Chance, K. V., De Mazière, M., Demoulin, P., Godin-Beekmann, S., Jones, N., Jucks, K., Keim, C., Kerzenmacher, T., Kuellmann, H., Kuttippurath, J., Iarlori, M., Liu, G. Y., Liu, Y., McDermid, I. S., Meijer, Y. J., Mencaraglia, F., Mikuteit, S., Oelhaf, H., Piccolo, C., Pirre, M., Raspollini, P., Ravegnani, F., Reburn, W. J., Redaelli, G., Remedios, J. J., Sembhi, H., Smale, D., Steck, T., Taddei, A., Varotsos, C., Vigouroux, C., Waterfall, A., Wetzel, G., and Wood, S.: Geophysical validation of MIPAS-ENVISAT operational ozone data, Atmos. Chem. Phys., 7, 4807-4867, doi:10.5194/acp-7-4807-2007, 2007.

Curtius, J., Weigel, R., Vössing, H.-J., Wernli, H., Werner, A., Volk, C.-M., Konopka, P., Krebsbach, M., Schiller, C., Roiger, A., Schlager, H., Dreiling, V., and Borrmann, S.: Observations of meteoric material and implications for aerosol nucleation in the winter Arctic lower stratosphere derived from insitu particle measurements, Atmos. Chem. Phys., 5, 3053-3069, doi:10.5194/acp-5-3053-2005, 2005.

Dinelli, B. M., Castelli, E., Carli, B., Del Bianco, S., Gai, M., Santurri, L., Moyna, B. P., Oldfield, M., Siddans, R., Gerber, D., Reburn, W. J., Kerridge, B. J., and Keim, C.: Technical Note: Measurement of the tropical UTLS composition in presence of clouds using millimetre-wave heterodyne spectroscopy, Atmos. Chem. Phys., 9, 1191-1207, doi:10.5194/acp-9-1191-2009, 2009.

Dörnbrack, A., Pitts, M. C., Poole, L. R., Orsolini, Y. J., Nishii, K., and Nakamura, H.: The 2009-2010 Arctic stratospheric winter general evolution, mountain waves and predictability of an operational weather forecast model, Atmos. Chem. Phys., 12, 36593675, doi:10.5194/acp-12-3659-2012, 2012.

Ewen, G. B. L., Grainger, R. G., Lambert, A., and Baran, A. J.: Infrared radiative transfer modelling in a $3 \mathrm{D}$ scattering cloudy atmosphere: Application to limb sounding measurements of cirrus, J. Quant. Spectrosc. Ra., 96, 45-74, 2005.
Fabian, B. and Borchers, R.: Halocarbons in the stratosphere, Nature, 294, 733-735, 1981.

Fischer, H. and Oelhaf, H.: Remote Sensing of vertical profiles of atmospheric trace constitutents with MIPAS limb-emission spectrometers, Appl. Opt., 35, 2787-2796, 1996.

Fischer, H., Birk, M., Blom, C., Carli, B., Carlotti, M., von Clarmann, T., Delbouille, L., Dudhia, A., Ehhalt, D., Endemann, M., Flaud, J. M., Gessner, R., Kleinert, A., Koopman, R., Langen, J., López-Puertas, M., Mosner, P., Nett, H., Oelhaf, H., Perron, G., Remedios, J., Ridolfi, M., Stiller, G., and Zander, R.: MIPAS: an instrument for atmospheric and climate research, Atmos. Chem. Phys., 8, 2151-2188, doi:10.5194/acp-8-2151-2008, 2008.

Flaud, J.-M., Piccolo, C., and Carli, B.: A spectroscopic database for MIPAS, in: Proceedings of the ENVISAT validation workshop, ESRIN, Italy, 2002.

Flaud, J.-M., Brizzi, G., Carlotti, M., Perrin, A., and Ridolfi, M.: MIPAS database: Validation of $\mathrm{HNO}_{3}$ line parameters using MIPAS satellite measurements, Atmos. Chem. Phys., 6, 5037-5048, doi:10.5194/acp-6-5037-2006, 2006.

Forman, M. L., Steel, W. H., and Vanasse, G.: Correction of asymmetric interferograms obtained in Fourier spectroscopy, J. Opt. Soc. Am., 56, 59-63, 1966.

Friedl-Vallon, F., Maucher, G., Seefeldner, M. Trieschmann, O., Kleinert, A., Lengel, A., Keim, C., Oelhaf, H., and Fischer, H.: Design and characterization of the balloon-borne Michelson Interferometer for Passive Atmospheric Sounding (MIPAS-B2), Appl. Opt., 43, 3335-3355, 2004.

Funke, B., Stiller, G. P., von Clarmann, T., Echle, G., and Fischer, $\mathrm{H}$.: $\mathrm{CO}_{2}$ line mixing in MIPAS limb emission spectra and its influence on retrieval of atmospheric parameters, J. Quant. Spectrosc. Ra., 59, 215-230, doi:10.1016/S0022-4073(97)00121-0, 1998.

Hofmann, D. J.: Increase in the Stratospheric Background Sulfuric Acid Aerosol Mass in the Past 10 Years, Science, 248, 996-1000, 1990.

Höpfner, M., Blom, C. E., von Clarmann, T., Fischer, H., Glatthor, N., Gulde, T., Hase, F., Keim, C., Kimmig, W., Lessenich, K., Piesch, C., Sartorius, C., and Stiller, G. P.: MIPAS-STR data analysis of APE-GAIA measurements, Paper presented in: IRS 2000: Current Problems in Atmospheric Radiation, edited by: Smith, W. L. and Timofeyev, Yu. M., A. Deepak Publishing, Hampton, Virginia, 1136-1139, 2000.

Höpfner, M., Blom, C. E., Echle, G., Glatthor, N., Hase, F., and Stiller, G.: Retrieval simulations for MIPAS-STR measurements, edited by: Smith, W. L., IRS 2000: Current Problems in Atmospheric Radiation; Proc. of the Internat. Radiation Symp., St. Petersburg, Russia, 24-29 July 2000 Hampton, Va., DEEPAK Publ., 2001.

Höpfner, M., Luo, B. P., Massoli, P., Cairo, F., Spang, R., Snels, M., Di Donfrancesco, G., Stiller, G., von Clarmann, T., Fischer, H., and Biermann, U.: Spectroscopic evidence for NAT, STS, and ice in MIPAS infrared limb emission measurements of polar stratospheric clouds, Atmos. Chem. Phys., 6, 1201-1219, doi:10.5194/acp-6-1201-2006, 2006.

Höpfner, M., von Clarmann, T., Fischer, H., Funke, B., Glatthor, N., Grabowski, U., Kellmann, S., Kiefer, M., Linden, A., Milz, M., Steck, T., Stiller, G. P., Bernath, P., Blom, C. E., Blumenstock, Th., Boone, C., Chance, K., Coffey, M. T., Friedl-Vallon, F., Griffith, D., Hannigan, J. W., Hase, F., Jones, N., Jucks, K. W., Keim, 
C., Kleinert, A., Kouker, W., Liu, G. Y., Mahieu, E., Mellqvist, J., Mikuteit, S., Notholt, J., Oelhaf, H., Piesch, C., Reddmann, T., Ruhnke, R., Schneider, M., Strandberg, A., Toon, G., Walker, K. A., Warneke, T., Wetzel, G., Wood, S., and Zander, R.: Validation of MIPAS $\mathrm{ClONO}_{2}$ measurements, Atmos. Chem. Phys., 7, 257-281, doi:10.5194/acp-7-257-2007, 2007.

Junge, C. E., Chagnon, C. W., and Manson, J. E.: A World-wide Stratospheric Aerosol Layer, Science, 133, 1478-1479, 1961.

Keim, C.: Entwicklung und Verifikation der Sichtlinienstabilisierung für MIPAS auf dem hochfliegenden Forschungsflugzeug M55 Geophysica, Wissenschafliche Berichte, FZKA 6729, Dissertation, Universität Karlsruhe, 2002.

Keim, C., Blom, C. E., von der Gathen, P., Gulde, T., Höpfner, M., Liu, G. Y., Oulanovsky, A., Piesch, C., Ravegnani, F., Sartorius, C., Schlager, H., and Volk, C. M.: Validation of MIPASENVISAT by correlative measurements of MIPAS-STR, Proc. ACVE-2 meeting, 3-7 May 2004, Frascati, Italy, 2004.

Keim, C., Liu, G. Y., Blom, C. E., Fischer, H., Gulde, T., Höpfner, M., Piesch, C., Ravegnani, F., Roiger, A., Schlager, H., and Sitnikov, N.: Vertical profile of peroxyacetyl nitrate (PAN) from MIPAS-STR measurements over Brazil in February 2005 and its contribution to tropical UT NO y partitioning, Atmos. Chem. Phys., 8, 4891-4902, doi:10.5194/acp-8-4891-2008, 2008.

Kimmig, W.: Das Abtastverfahren der Interferogramme des flugzeuggetragenen Fourierspektrometers MIPAS-STR, Wissenschaftliche Berichte, FZKA 6665, Dissertation, Universität Karlsruhe, 2001.

Kleinert, A.: Correction of detector nonlinearity for the balloonborne Michelson Interferometer for Passive Atmospheric Sounding, Appl. Opt., 45, 425-431, 2006.

Kleinert, A. and Trieschmann, O.: Phase determination for a Fourier transform infrared spectrometer in emission mode, Appl. Opt., 46, 2307-2319, 2007.

Manuilova, R. O., Gusev, O. A., Kutepov, A. A., von Clarmann, T., Oelhaf, H., Stiller, G. P., Wegner, A., Lopez-Puertas, M., MartinTorres, F. J., Zaragoza, G., and Flaud, J.-M.: Modelling of nonLTE limb spectra of i.r. ozone bands for the MIPAS space experiment, J. Quant. Spectrosc. Ra., 59, 405-422, doi:10.1016/S00224073(97)00120-9, 1998.

Moore, D. P., Waterfall, A. M., and Remedios, J. J.: The potential for radiometric retrievals of halocarbon concentrations from the MIPAS-E instrument, Adv. Space Res., 37, 2238-2246, 2006.

Murphy, D. M., Thomson, D. S., and Mahoney, M. J.: In-Situ Measurements of Organics, Meteoritic Material, Mercury, and Other Elements in Aerosols at 5 to 19 Kilometers, Science, 282, 16641669, 1998.

Norton, H. and Beer, R.: New apodizing functions for Fourier spectrometry, J. Opt. Soc. Am., 66, 259-264, (Errata J. Opt. Soc. Am., 67, 419, 1977), 1976.

Peter, T.: Microphysics and heterogeneous chemistry of polar stratospheric clouds, Ann. Rev. Phys. Chem., 48, 785-822, 1997.

Phillips, C.: A Technique for the Numerical Solution of Certain Integral Equations of the First Kind, J. Assoc. Comput. Math., 9, 84-97, 2003.

Piesch, C., Gulde, T., Sartorius, C., Friedl-Vallon, F., Seefeldner, M., Wölfel, M., Blom, C. E., and Fischer, H.: Design of a MIPAS instrument for high-altitude aircraft, Proc. of the 2nd Internat. Airborne Remote Sensing Conference and Exhibition, ERIM, Ann Arbor, MI, Vol. II, 199-208, 1996.
Purser, R. J. and Huang, H.-L.: Estimating effective data density in a satellite retrieval or an objective analysis, J. Appl. Meteorol., 32, 1092-1107, 1993.

Remedios, J. J., Leigh, R. J., Waterfall, A. M., Moore, D. P., Sembhi, H., Parkes, I., Greenhough, J., Chipperfield, M.P., and Hauglustaine, D.: MIPAS reference atmospheres and comparisons to V4.61/V4.62 MIPAS level 2 geophysical data sets, Atmos. Chem. Phys. Discuss., 7, 9973-10017, doi:10.5194/acpd-79973-2007, 2007.

Riediger, O., Volk, C. M., Strunk, M., and Schmidt, U.: HAGAR - A new in- situ tracer instrument for stratospheric balloons and high altitude aircraft, Eur. Comm. Air Pollut. Res. Report, 73, 727-730, 2000.

Rodgers, C. D.: Inverse Methods for Atmospheric Sounding: Theory and Practice, Vol. 2 of Series on Atmospheric, Oceanic and Planetary Physics, edited by: Taylor, F. W., World Scientific, 2000.

Schneider, M., Hase, F., Blavier, J.-F., Toon, G.C., and Leblanc, T.: An empirical study on the importance of a speed-dependent Voigt line shape model for tropospheric water vapor profile remote sensing, J. Quant. Spectrosc. Ra., 112, 465-474, doi:10.1016/j.jqsrt.2010.09.008, 2011.

Shur, G. N., Yushkov, V. A., Drynkov, A. V., Fadeeva, G. V., and Potertikova, G. A.: Study of Thermodynamics of the Stratosphere at High Latitudes of the Northern Hemisphere on the M55 Geofizika Flying Laboratory, Russ. Meteorol. Hydrol., 8, 4353, 2006.

Sitnikov, N. M., Yushkov, V. A., Afchine, A. A., Korshunov, L. I., Astakhov, V. I., Ulanovskii, A. E., Krämer, M., Mangold, A., Schiller, C., and Ravegnani, F.: The FLASH instrument for water vapor measurements on board the high-altitude airplane, Instrum. Exp. Tech., 50, 113-121, doi:10.1134/S0020441207010174, 2007.

Spang, R., Eidmann, G., Riese, M., Offermann, D., Preusse, P., Pfister, L., and Wang, P.-H.: CRISTA observations of cirrus clouds around the tropopause, J. Geophys. Res., 107, 8174, doi:10.1029/2001JD000698, 2002.

Spang, R., Remedios, J. J., and Barkley, M. P.: Colour indices for the detection and differentiation of cloud types in infra-red limb emission spectra, Adv. Space Res., 33, 1041-1047, 2004.

Spang, R., Hoffmann, L., Kullmann, A., Olschewski, F., Preusse, P., Knieling, P., Schroeder, S., Stroh, F., Weigel, K., and Riese, M.: High resolution limb observations of clouds by the CRISTA-NF experiment during the $\mathrm{SCOUT}_{3} \mathrm{O}_{3}$ tropical aircraft campaign, J. Adv. Space, Res., 42, 1765-1775, doi:10.1016/j.asr.2007.09.036, 2008.

Steck, T.: Methods for determining regularization for atmospheric retrieval problems, Appl. Opt., 41, 1788-1797, 2002.

Stiller, G. P. (Ed.) with contributions from von Clarmann, T., Dudhia, A., Echle, G., Funke, B., Glatthor, N., Hase, F., Höpfner, M., Kellmann, S., Kemnitzer, H., Kuntz, M., Linden, A., Linder, M., Stiller, G. P., and Zorn, S.: The Karlsruhe Optimized and Precise Radiative Transfer Algorithm (KOPRA), Wissenschaftliche Berichte, FZKA 6487, Forschungszentrum Karlsruhe, 2000.

Stiller, G. P., von Clarmann, T., Funke, B., Glatthor, N., Hase, F., Höpfner, M., and Linden, A.: Sensitivity of trace gas abundances retrievals from infrared limb emission spectra to simplifying approximations in radiative transfer modelling, J. Quant. Spectrosc. Ra., 72, 249-280, doi:10.1016/S0022-4073(01)00123-6, 2002. 
Tikhonov, A.: On the Solution of Incorrectly Stated Problems and a Method of Regularisation, Dokl. Acad. Nauk SSSR, 151, 501504, 1963.

Trieschmann, O., Friedl-Vallon, F., Lengel, A., Oelhaf, H., Wetzel, G., and Fischer H.: An advanced phase correction approach to obtain radiometric calibrated spectra of the optically well balanced balloon borne Fourier Transform Spectrometer MIPASB2, Optical Spectroscopic Techniques and Instrumentation for Atmospheric and Space Research III, edited by: Larar, A. M., SPIE, 3756, 17-24, 1999.

Ulanovsky, A. E., Yushkov, V. A., Sitnikov, N. M., and Ravegnani, F.: The FOZAN-II Fast-Response Chemiluminescent Airborne Ozone Analyzer, Instrum. Exp. Tech., 44, 249-256, 2001.

Ungermann, J., Kalicinsky, C., Olschewski, F., Knieling, P., Hoffmann, L., Blank, J., Woiwode, W., Oelhaf, H., Hösen, E., Volk, C. M., Ulanovsky, A., Ravegnani, F., Weigel, K., Stroh, F., and Riese, M.: CRISTA-NF measurements with unprecedented vertical resolution during the RECONCILE aircraft campaign, Atmos. Meas. Tech. Discuss., 4, 6915-6967, doi:10.5194/amtd-46915-2011, 2011.

von Clarmann, T.: Zur Fernerkundung der Erdatmosphäre mittels Infrarotspektroskopie: Rekonstruktionstheorie und Anwendung, Wissenschaftliche Berichte, FZKA 6928, Forschungszentrum Karlsruhe, 2003.
Wagner, G. and Birk, M.: New infrared spectroscopic database for chlorine nitrate, J. Quant. Spectrosc. Ra., 82, 443-460, 2003.

Wang, D. Y., Höpfner, M., Blom, C. E., Ward, W. E., Fischer, H., Blumenstock, T., Hase, F., Keim, C., Liu, G. Y., Mikuteit, S., Oelhaf, H., Wetzel, G., Cortesi, U., Mencaraglia, F., Bianchini, G., Redaelli, G., Pirre, M., Catoire, V., Huret, N., Vigouroux, C., De Mazière, M., Mahieu, E., Demoulin, P., Wood, S., Smale, D., Jones, N., Nakajima, H., Sugita, T., Urban, J., Murtagh, D., Boone, C. D., Bernath, P. F., Walker, K. A., Kuttippurath, J., Kleinböhl, A., Toon, G., and Piccolo, C.: Validation of MIPAS $\mathrm{HNO}_{3}$ operational data, Atmos. Chem. Phys., 7, 4905-4934, doi:10.5194/acp-7-4905-2007, 2007.

Werner, A., Volk, C. M., Ivanova, E. V., Wetter, T., Schiller, C., Schlager, H., and Konopka, P.: Quantifying transport into the Arctic lowermost stratosphere, Atmos. Chem. Phys., 10, 11623 11639, doi:10.5194/acp-10-11623-2010, 2010.

Wetzel, G., Oelhaf, H., Ruhnke, R., Friedl-Vallon, F., Kleinert, A., Kouker, W., Maucher, G., Reddmann, T., Seefeldner, M., Stowasser, M., Trieschmann, O., von Clarmann, T., and Fischer, $\mathrm{H}$.: $\mathrm{NO}_{y}$ partitioning and budget and its correlation with $\mathrm{N}_{2} \mathrm{O}$ in the Arctic vortex and in summer midlatitudes in 1997, J. Geophys. Res., 107, 4280, doi:10.1029/2001JD000916, 2002. 